\title{
On the existence of coupled best proximity point and best proximity point for Suzuki type $\alpha^{+}-\theta$-proximal multivalued mappings
}

\author{
Haiming Liü ${ }^{\mathrm{a}, *}$, Xiaoming Fan ${ }^{\mathrm{b}}$, Lixu Yan ${ }^{\mathrm{c}}$, Zhigang Wang ${ }^{\mathrm{b}, *}$ \\ a School of Mathematics, Mudanjiang Normal University, Mudanjiang, 157011, P. R. China. \\ ${ }^{b}$ School of Mathematical Sciences, Harbin Normal University, Harbin, 150025, P. R. China. \\ ${ }^{c}$ Department of Mathematics, Harbin Institute of Technology, Harbin, 150001, P. R. China.
}

Communicated by Y. J. Cho

\begin{abstract}
Following the study for the best proximity points of the Suzuki type $\alpha^{+}-\theta$-proximal single-valued mappings given by Hussain et al., we deal with the Suzuki type $\alpha^{+}-\theta$-proximal multivalued mappings satisfying generalized conditions of existence, some novel existence results of best proximity point and coupled best proximity points are established. Our results improve and extend some recent results in the previous work. (C)2017 All rights reserved.
\end{abstract}

Keywords: Suzuki type $\alpha^{+}-\theta$-proximal multivalued mappings, coupled best proximity point, best proximity point. 2010 MSC: 47H09, 54H25.

\section{Introduction}

Employing the limit of Picard iterates, the classical Banach's contractive principal verifies the existence and uniqueness of the fixed point equation $T x=x$, where $T$ is a self-mapping on a complete metric space $X$ and $x \in X$. However, if $T$ a non-self-mapping from $A$ to $B$, where $A, B$ are two nonempty subsets of $X$, then $d(x, T x) \geqslant \operatorname{dist}(A, B)$, the equation $T x=x$ has no solution, naturally, in this case, one usually hopes to find an element $x \in A$ closing $T x$ such that the error $d(x, T x)$ is minimal, that is, to consider the existence of optimal approximate solutions for such fixed point equations. A problem arises: Is there such point satisfying $d(x, T x)=\operatorname{dist}(A, B)$ ?

In other words, does an element $x \in A$ with the minimum $\operatorname{dist}(A, B)$ error $d(x, T x)$ exist?

For the sake of clearness in the introduction, we give some notations which will be used in the sequel: Let $(X, d)$ be a metric space and $A, B \subset X$,

$$
\begin{aligned}
\operatorname{dist}(A, B) & =\inf \{d(x, y): x \in A, y \in B\}, D(x, B)=\inf \{d(x, y): y \in B\}, \\
A_{0} & =\{a \in A: d(a, b)=\operatorname{dist}(A, B) \text { for some } b \in B\}, \\
B_{0} & =\{b \in B: d(a, b)=\operatorname{dist}(A, B) \text { for some } a \in A\} .
\end{aligned}
$$

For any two nonempty sets $A$ and $B$ in a metric space $(X, d)$, the point $a \in A_{0}$ is called a best proximity

\footnotetext{
*Corresponding author

Email addresses: liuhm468@nenu.edu.cn (Haiming Liu), fanxm093@163.com (Xiaoming Fan), luckyyan1990@163.com (Lixu Yan), wangzg2003205@163.com (Zhigang Wang)

doi:10.22436/jnsa.010.04.41
} 
point of the mapping $T: A \rightarrow B$, if $d(a, T a)=\operatorname{dist}(A, B)$. In 1969, addressing the problem above, Fan [5] established a classical best approximation theorem. Afterward, the existence and convergence of best proximity points have become interesting topics of optimization theory which recently attracted the attention of many authors, they devote themselves to the investigate of best proximity points of mappings in a variety of settings and have given many extended results for Fan's Theorem $[2-4,8,10,14-$ $19,21,22,24,26]$. These extensions mainly lie in two aspects, the one comes from the development of spaces, the other comes from the change of mappings, that is, under various of contractive conditions for certain spaces, the proximity points are considered. Especially, best proximity theorems also serve as a natural generalization of fixed point theorems, if the mapping under consideration is a self-mapping. If we take $T$ as $T: A \times A \rightarrow B$, that is, $T$ is a two-variable mapping, such pair point $(x, y)$ satisfying $d(x, T(x, y))=d(y, T(y, x))=\operatorname{dist}(A, B)$ is called coupled best proximity point. As a result, some best proximity results were further generalized into coupled best proximity results when considering the two-variable mappings. If ones extend the class of single-valued mappings to the class of multivalued mappings $T: A \rightarrow 2^{B} \backslash\{\emptyset\}$ and moreover, the class of one-variable multivalued mappings to the class of two-variable multivalued mappings $T: A \times A \rightarrow 2^{B} \backslash\{\emptyset\}$, then the situation is getting more complicated and the results of proximity points are more generalized. In this paper, we focus our attentions on the study of one-variable and two-variable multivalued mappings.

Some problems of fixed points of either single-valued or multivalued mappings involving $\alpha$-admissible have become a hotspot research since Samet et al. [20] introduced the notion of $\alpha$-admissible in 2012, for example, following Samet's definition, Latif et al. [9] defined the concept of $(\alpha, \psi)$-Meir-Keeler selfmappings. Redjel et al. [13] introduced a concept of $(\alpha, \psi)$-Meir-Keeler-Khan mappings, we also defined the class of $(\alpha, \psi)$-Meir-Keeler-Khan multivalued mappings recently [25]. Hussain et al. [6] introduced the concept of proximal $\alpha^{+}$-admissible and introduced new Suzuki type contractions and proved new best proximity results for these contractions in the setting of a metric space, they dealt with best proximity theorems for Suzuki contractions involving the $\theta$ function which was introduced by Jleli and Samet [7]. Sintunavarat and Kumam [23] introduced the concept of coupled best proximity point and proved the coupled best proximity theorem for involving cyclic contractions. Recently, Nantadilok [11] established coupled best proximity point theorems for multivalued mappings via $\alpha$-admissible notion and $\psi$ function.

In this paper, we devote ourselves to study the existence of coupled best proximity point and best proximity point of the new types of proximal multivalued mappings. In Section 2, we present some preliminaries and recall the results for $\alpha^{+}-\theta$-proximal single-valued mappings. In Section 3, inspired and motivated by Hussain et al. [6], Sintunavarat et al. [23], Jleli et al. [7], and Nantadilok [11], we define the notion of $\alpha^{+}-\theta$-proximal multivalued mapping for one-variable and two-variable and explore the best proximity point theorem and the coupled best proximity point theorem for the class of $\alpha^{+}-\theta$-proximal multivalued mappings in this paper. The existence theorems of coupled best proximity points for twovariable $\alpha^{+}-\theta$-proximal multivalued mappings with the hypothesis of continuity or regularity and the best proximity point results for one-variable $\alpha^{+}-\theta$-proximal multivalued mappings with the hypothesis of continuity or regularity are established, respectively. These more generalized results extend and generalize the main results of Hussain et al., Nantadilok, and Jleli et al. in the literatures [6, 7, 11, 23, 24]. We also provide an example to show the generality and effectiveness of our results.

\section{Preliminaries}

As the preliminaries, we review some definitions (see $[6,9,12]$ and references therein).

Definition 2.1 ([6]). Let $T$ be a self-mapping on a nonempty set $X$ and $\alpha: X \times X \rightarrow[-\infty,+\infty)$ be a mapping. The mapping $T$ is said to be proximal $\alpha^{+}$-admissible, if the following condition holds:

$$
\left.\begin{array}{r}
\alpha(x, y) \geqslant 0, \\
d\left(u_{1}, T x_{1}\right)=\operatorname{dist}(A, B), \\
d\left(u_{2}, T x_{2}\right)=\operatorname{dist}(A, B),
\end{array}\right\} \Rightarrow \alpha\left(u_{1}, u_{2}\right) \geqslant 0,
$$


for all $x_{1}, x_{2}, u_{1}, u_{2} \in A$.

Definition 2.2 ([12]). For nonempty subsets $A, B$ of a metric space $(X, d)$ with $A_{0} \neq \emptyset$, we say the pair $(A, B)$ satisfies

(a) the P-property, if

$$
\left.\begin{array}{l}
d\left(x_{1}, y_{1}\right)=\operatorname{dist}(A, B), \\
d\left(x_{2}, y_{2}\right)=\operatorname{dist}(A, B),
\end{array}\right\} \Rightarrow d\left(x_{1}, x_{2}\right)=d\left(y_{1}, y_{2}\right)
$$

for all $x_{1}, x_{2} \in A_{0}$ and $y_{1}, y_{2} \in B_{0}$;

(b) the weak P-property, if for any $x_{1}, x_{2} \in A_{0}$ and $y_{1}, y_{2} \in B_{0}$,

$$
\left.\begin{array}{l}
d\left(x_{1}, y_{1}\right)=\operatorname{dist}(A, B), \\
d\left(x_{2}, y_{2}\right)=\operatorname{dist}(A, B),
\end{array}\right\} \Rightarrow d\left(x_{1}, x_{2}\right) \leqslant d\left(y_{1}, y_{2}\right)
$$

Jleli and Samet [7] introduced a new type of contractive maps related to $\theta$ functions and established some fixed point results for such mappings. In this section, we deal with coupled best proximity theorems and best proximity theorems for Suzuki type contractions involving the $\theta$ functions.

We denote by $\Theta$ the family of all functions $\theta:(0, \infty) \rightarrow(1, \infty)$ satisfying the following conditions:

$\left(\Theta_{1}\right) \theta$ is nondecreasing;

$\left(\Theta_{2}\right)$ for each sequence $\left\{\beta_{n}\right\}_{n=0}^{\infty} \subset(0, \infty), \lim _{n \rightarrow \infty} \beta_{n}=0^{+}$if and only if $\lim _{n \rightarrow \infty} \theta\left(\beta_{n}\right)=1$;

$\left(\Theta_{3}\right)$ there exist $r \in(0,1)$ and $\ell \in(0, \infty]$ such that $\lim _{\beta \rightarrow 0^{+}} \frac{\theta(\beta)-1}{\beta^{r}}=\ell$.

Definition $2.3([6])$. Let $(X, d)$ be a metric space. $T: A \rightarrow B$ is called a Suzuki type $\alpha^{+}-\theta$-proximal mapping, if there exist two functions $\theta \in \Theta$ and $\alpha: X \times X \rightarrow[-\infty,+\infty)$ such that for all $x, y \in A$,

$$
\frac{1}{2} d^{*}(x, T x) \leqslant d(x, T x) \Rightarrow \alpha(x, y)+\theta(d(T x, T y)) \leqslant[\theta(M(x, y))]^{k},
$$

where

$$
d^{*}(x, T x)=d(x, T x)-\operatorname{dist}(A, B),
$$

and

$$
M(x, y)=\max \left\{d(x, y), \frac{d(x, T x)+d(y, T y)}{2}-\operatorname{dist}(A, B), \frac{d(x, T y)+d(y, T x)}{2}-\operatorname{dist}(A, B)\right\} .
$$

Hussian et al. [6] established existence theorem for best proximity points of Suzuki type $\alpha^{+}-\theta$ proximal mappings with continuity assumption or regularity on the mappings.

Theorem 2.4 ([6]). Suppose $A$ and $B$ are nonempty closed subsets of a metric space $(X, d)$ with $A_{0} \neq \emptyset$. Let $\mathrm{T}: \mathrm{A} \rightarrow \mathrm{B}$ satisfy (2.1) together with the following assertions:

(i) $\mathrm{T}\left(\mathrm{A}_{0}\right) \subseteq \mathrm{B}_{0}$ and $(\mathrm{A}, \mathrm{B})$ satisfies the weak P-property;

(ii) $\mathrm{T}$ is proximal $\alpha^{+}$-admissible;

(iii) there exist $\mathrm{x}_{0}, \mathrm{x}_{1} \in \mathrm{A}_{0}$ such that

$$
\mathrm{d}(\mathrm{x}, \mathrm{Tx})=\operatorname{dist}(\mathrm{A}, \mathrm{B}) \text { and } \alpha\left(\mathrm{x}_{0}, \mathrm{x}_{1}\right) \geqslant 0 ;
$$

(iv) T is continuous; or

(v) $A$ is $\alpha$-regular, that is, if $\left\{x_{n}\right\}$ is a sequence in $A$ such that $\alpha\left(x_{n}, x_{n+1}\right) \geqslant 0$ and $x_{n} \rightarrow x \in A$ as $n \rightarrow \infty$, then $\alpha\left(x_{n}, x\right) \geqslant 0$, for all $n \in \mathbb{N}$.

Then there exists $x^{*} \in A_{0}$ such that $d\left(x^{*}, T x^{*}\right)=\operatorname{dist}(A, B)$. 


\section{Coupled best proximity points and best proximity points for Suzuki type $\alpha^{+}-\theta$-proximal mappings}

In the sequel, $\mathbb{N}$ denotes the set of all nonnegative integers, $\operatorname{Bpp}(\mathrm{T})$ denotes the set of best proximity points of $T, C B p p(T)$ denotes the set of coupled best proximity points of $T$, and $\operatorname{CL}(X)$ denotes the family of nonempty closed subsets of $X$.

For any $A, B \in \mathcal{C} L(X)$, let the mapping $H(\cdot, \cdot)$ be the generalized Hausdorff distance with respect to $d$ defined by

$$
H(A, B)= \begin{cases}\max \left\{\sup _{x \in A} \operatorname{dist}(x, B), \sup _{y \in B} \operatorname{dist}(y, A)\right\} & \text { if it exists, } \\ \infty & \text { otherwise. }\end{cases}
$$

Definition $3.1([1,11])$. An element $x^{*} \in A$ is said to be the best proximity point of a multivalued nonself-mapping $T: A \rightarrow 2^{B} \backslash\{\emptyset\}$, if $D\left(x^{*}, T x^{*}\right)=\operatorname{dist}(A, B)$.

Definition 3.2. Let $(X, d)$ be a metric space and $A, B \in \mathcal{C L}(X)$. A multivalued mapping $T: A \rightarrow 2^{B} \backslash\{\emptyset\}$ is called proximal $\alpha^{+}$-admissible, if there exists a mapping $\alpha: A \times A \rightarrow[-\infty,+\infty)$ such that for any $x_{1}, x_{2}, u_{1}, u_{2} \in A$ and $y_{1} \in T x_{1}, y_{2} \in T x_{2}$,

$$
\left.\begin{array}{r}
\alpha\left(x_{1}, x_{2}\right) \geqslant 0, \\
d\left(u_{1}, y_{1}\right)=\operatorname{dist}(A, B), \\
d\left(u_{2}, y_{2}\right)=\operatorname{dist}(A, B),
\end{array}\right\} \Rightarrow \alpha\left(u_{1}, u_{2}\right) \geqslant 0
$$

Definition 3.3. Let $(X, d)$ be a metric space and $A, B \in \mathcal{C} L(X)$. A mapping $T: A \times A \rightarrow 2^{B} \backslash\{\emptyset\}$ is called proximal $\alpha^{+}$-admissible, if there exists a mapping $\alpha: A \times A \rightarrow[-\infty,+\infty)$ such that for any $x_{1}, x_{2}, w_{1}, w_{2}, w_{1}^{\prime}, w_{2}^{\prime}, y_{1}, y_{2} \in A$ and $u_{1} \in T\left(x_{1}, y_{1}\right), u_{2} \in T\left(x_{2}, y_{2}\right), v_{1} \in T\left(y_{1}, x_{1}\right), v_{2} \in T\left(y_{2}, x_{2}\right)$,

$$
\left.\begin{array}{r}
\alpha\left(x_{1}, x_{2}\right) \geqslant 0, \\
d\left(w_{1}, u_{1}\right)=\operatorname{dist}(A, B), \\
d\left(w_{2}, u_{2}\right)=\operatorname{dist}(A, B),
\end{array}\right\} \Rightarrow \alpha\left(w_{1}, w_{2}\right) \geqslant 0,
$$

and

$$
\left.\begin{array}{r}
\alpha\left(y_{1}, y_{2}\right) \geqslant 0, \\
d\left(w_{1}^{\prime}, v_{1}\right)=\operatorname{dist}(A, B), \\
d\left(w_{2}^{\prime}, v_{2}\right)=\operatorname{dist}(A, B),
\end{array}\right\} \Rightarrow \alpha\left(w_{1}^{\prime}, w_{2}^{\prime}\right) \geqslant 0
$$

Definition $3.4([11])$. Let $(X, d)$ be a complete metric space and $A, B \in \operatorname{CL}(X)$. An element $\left(x^{*}, y^{*}\right) \in$ $(A \times A)$ is said to be the coupled best proximity point of a multivalued mapping $T: A \times A \rightarrow \operatorname{CL}(B)$, if $\mathrm{D}\left(\mathrm{x}^{*}, \mathrm{~T}\left(\mathrm{x}^{*}, \mathrm{y}^{*}\right)\right)=\operatorname{dist}(A, B)$ and $\mathrm{D}\left(\mathrm{y}^{*}, \mathrm{~T}\left(\mathrm{y}^{*}, x^{*}\right)\right)=\operatorname{dist}(A, B)$.

Lemma 3.5 ([11]). Let $\mathrm{B}$ be nonempty closed subsets of a metric space $(\mathrm{X}, \mathrm{d})$. Then, for each $\mathrm{x} \in \mathrm{X}$ with $\mathrm{D}(\mathrm{x}, \mathrm{B})>0$ and $\mathrm{q}>1$, there exists an element $\mathrm{b} \in \mathrm{B}$ such that

$$
\mathrm{d}(x, \mathrm{~b})<\mathrm{qD}(x, \mathrm{~B}) .
$$

Definition 3.6. Let $\mathrm{T}: \mathrm{A} \rightarrow \mathrm{CL}(\mathrm{B})$ be a mapping on a metric space $(X, d)$. $T$ is called a one-variable Suzuki type $\alpha^{+}-\theta$-proximal multivalued mapping, if there exist two functions $\theta \in \Theta, \alpha: X \times X \rightarrow[-\infty,+\infty)$, and $0<k<1, q>1$ such that for all $x, y \in A$,

$$
\frac{1}{2} D^{*}(x, T x) \leqslant d(x, y) \Rightarrow \alpha(x, y)+\theta(q H(T x, T y)) \leqslant\left[\theta(M(x, y)]^{k},\right.
$$

when $H(T x, T y)>0$, where

$$
D^{*}(x, T x)=D(x, T x)-\operatorname{dist}(A, B)
$$

and

$$
M(x, y)=\max \left\{d(x, y), \frac{D(x, T x)+D(y, T y)}{2}-\operatorname{dist}(A, B), \frac{D(x, T y)+D(y, T x)}{2}-\operatorname{dist}(A, B)\right\} .
$$


Definition 3.7. Let $T: A \times A \rightarrow \mathcal{C L}(B)$ be a mapping on a metric space $(X, d)$. $T$ is called a two-variable Suzuki type $\alpha^{+}-\theta$-proximal multivalued mapping, if there exist two functions $\theta \in \Theta, \alpha: X \times X \rightarrow$ $[-\infty,+\infty)$, and $0<k<1, q>1$ such that for all $x, y, x^{\prime}, y^{\prime} \in A$,

$$
\left.\begin{array}{l}
\frac{1}{2} D^{*}\left(x, T\left(x, x^{\prime}\right)\right) \leqslant d(x, y), \\
\frac{1}{2} D^{*}\left(x^{\prime}, T\left(x^{\prime}, x\right)\right) \leqslant d\left(x^{\prime}, y^{\prime}\right), \\
d(x, y)=d\left(x^{\prime}, y^{\prime}\right)=0, \text { or } \\
d(x, y)>0, d\left(x^{\prime}, y^{\prime}\right)>0,
\end{array}\right\} \Rightarrow \alpha(x, y)+\theta\left(q H\left(T\left(x, x^{\prime}\right), T\left(y, y^{\prime}\right)\right)\right) \leqslant\left[\theta\left(M\left(x, y, x^{\prime}, y^{\prime}\right)\right)\right]^{k},
$$

when $H\left(T\left(x, x^{\prime}\right), T\left(y, y^{\prime}\right)\right)>0$ and $H\left(T\left(x^{\prime}, x\right), T\left(y^{\prime}, y\right)\right)>0$, where

$$
\mathrm{D}^{*}\left(x, \mathrm{~T}\left(x, x^{\prime}\right)\right)=\mathrm{D}\left(x, \mathrm{~T}\left(x, x^{\prime}\right)\right)-\operatorname{dist}(\mathrm{A}, \mathrm{B}),
$$

and

$$
\begin{aligned}
& M\left(x, y, x^{\prime}, y^{\prime}\right)=\max \left\{d(x, y), \frac{D\left(x, T\left(x, x^{\prime}\right)\right)+D\left(y, T\left(y, y^{\prime}\right)\right)}{2}-\operatorname{dist}(A, B),\right. \\
& \left.\frac{D\left(y, T\left(x, x^{\prime}\right)\right)+D\left(x, T\left(y, y^{\prime}\right)\right)}{2}-\operatorname{dist}(A, B)\right\} \text {. }
\end{aligned}
$$

The following coupled best proximity result can be stated:

Theorem 3.8. Let $(X, d)$ be a complete metric space and $A, B \in \mathcal{C L}(X)$ with $A_{0} \neq \emptyset . \theta \in \Theta$ and $T: A \times A \rightarrow \operatorname{CL}(B)$ be a two-variable Suzuki type $\alpha^{+}-\theta$ - proximal multivalued mapping. Suppose that the following conditions hold:

(i) $\mathrm{T}(\mathrm{x}, \mathrm{y}) \subseteq \mathrm{B}_{0}$ for $(\mathrm{x}, \mathrm{y}) \in \mathrm{A}_{0} \times \mathrm{A}_{0}$ and $(\mathrm{A}, \mathrm{B})$ satisfies the weak P-property;

(ii) $\mathrm{T}$ is $\alpha^{+}$-proximal admissible;

(iii) there exist elements $\left(x_{0}, y_{0}\right),\left(x_{1}, y_{1}\right) \in\left(A_{0} \times A_{0}\right)$ and $u_{1} \in T\left(x_{0}, y_{0}\right), v_{1} \in T\left(y_{0}, x_{0}\right)$ such that

$$
\begin{aligned}
& d\left(x_{1}, u_{1}\right)=\operatorname{dist}(A, B), \quad \alpha\left(x_{0}, x_{1}\right) \geqslant 0, \\
& d\left(y_{1}, v_{1}\right)=\operatorname{dist}(A, B), \quad \alpha\left(y_{0}, y_{1}\right) \geqslant 0 ;
\end{aligned}
$$

(iv) if $\mathrm{D}(\mathrm{x}, \mathrm{T}(\mathrm{x}, \mathrm{y}))=0$ or $\mathrm{D}(\mathrm{y}, \mathrm{T}(\mathrm{y}, \mathrm{x}))=0$, for any $\mathrm{x}, \mathrm{y} \in \mathrm{A}_{0}$, then $\mathrm{D}(\mathrm{x}, \mathrm{T}(\mathrm{x}, \mathrm{y}))=\mathrm{D}(\mathrm{y}, \mathrm{T}(\mathrm{y}, \mathrm{x}))=0$;

(v) T is continuous; or

(vi) if $\left\{x_{n}\right\}_{n=0}^{\infty}$ is a sequence in $A$ such that $\alpha\left(x_{n}, x_{n+1}\right) \geqslant 0$ and $x_{n} \rightarrow x^{*} \in A$ as $n \rightarrow \infty$, then $\alpha\left(x_{n}, x^{*}\right) \geqslant 0$ for all $\mathrm{n} \in \mathbb{N}$.

Then $\mathrm{CBpp}(\mathrm{T})$ is nonempty.

Proof. By condition (iii), there exist elements $\left(x_{0}, y_{0}\right),\left(x_{1}, y_{1}\right) \in\left(A_{0} \times A_{0}\right)$ and $u_{1} \in T\left(x_{0}, y_{0}\right), v_{1} \in T\left(y_{0}, x_{0}\right)$ such that

$$
\begin{aligned}
& d\left(x_{1}, u_{1}\right)=\operatorname{dist}(A, B), \quad \alpha\left(x_{0}, x_{1}\right) \geqslant 0, \\
& d\left(y_{1}, v_{1}\right)=\operatorname{dist}(A, B), \quad \alpha\left(y_{0}, y_{1}\right) \geqslant 0 .
\end{aligned}
$$

We consider the following four cases:
(a) $\mathrm{u}_{1} \in \mathrm{T}\left(\mathrm{x}_{1}, \mathrm{y}_{1}\right), v_{1} \notin \mathrm{T}\left(\mathrm{y}_{1}, \mathrm{x}_{1}\right)$,
(b) $\mathrm{u}_{1} \notin \mathrm{T}\left(\mathrm{x}_{1}, \mathrm{y}_{1}\right), v_{1} \in \mathrm{T}\left(\mathrm{y}_{1}, \mathrm{x}_{1}\right)$,
(c) $u_{1} \in T\left(x_{1}, y_{1}\right), v_{1} \in T\left(y_{1}, x_{1}\right)$,
(d) $\mathrm{u}_{1} \notin \mathrm{T}\left(\mathrm{x}_{1}, \mathrm{y}_{1}\right), v_{1} \notin \mathrm{T}\left(\mathrm{y}_{1}, \mathrm{x}_{1}\right)$.

Case (a). Since $u_{1} \in A_{0}, T\left(x_{1}, y_{1}\right) \subseteq B_{0}$, then

$$
0=D\left(u_{1}, T\left(x_{1}, y_{1}\right)\right) \geqslant \operatorname{dist}\left(A_{0}, B_{0}\right) \geqslant \operatorname{dist}(A, B) \geqslant 0,
$$


hence,

$$
\mathrm{D}\left(\mathrm{u}_{1}, \mathrm{~T}\left(\mathrm{x}_{1}, \mathrm{y}_{1}\right)\right)=\operatorname{dist}(\mathrm{A}, \mathrm{B})=0
$$

From (3.2), we get

$$
\mathrm{d}\left(\mathrm{x}_{1}, \mathrm{u}_{1}\right)=0, \mathrm{~d}\left(\mathrm{y}_{1}, v_{1}\right)=0,
$$

that is, $x_{1}=u_{1}, y_{1}=v_{1}$, hence, applying (iv), we have

$$
\begin{aligned}
& \mathrm{D}\left(\mathrm{x}_{1}, \mathrm{~T}\left(\mathrm{x}_{1}, \mathrm{y}_{1}\right)\right)=\mathrm{D}\left(\mathrm{u}_{1}, \mathrm{~T}\left(\mathrm{x}_{1}, \mathrm{y}_{1}\right)\right)=\operatorname{dist}(\mathrm{A}, \mathrm{B})=0, \\
& \mathrm{D}\left(\mathrm{y}_{1}, \mathrm{~T}\left(\mathrm{y}_{1}, \mathrm{x}_{1}\right)\right)=\mathrm{D}\left(v_{1}, \mathrm{~T}\left(\mathrm{y}_{1}, x_{1}\right)\right)=\operatorname{dist}(A, \mathrm{~B})=0 .
\end{aligned}
$$

On the other hand, since $v_{1} \notin T\left(y_{1}, x_{1}\right)$ and $T\left(y_{1}, x_{1}\right) \in \mathcal{C L}(B)$, therefore $D\left(y_{1}, T\left(y_{1}, x_{1}\right)\right)=D\left(v_{1}, T\left(y_{1}, x_{1}\right)\right)>$ 0 , which contradicts to $\mathrm{D}\left(\mathrm{y}_{1}, \mathrm{~T}\left(\mathrm{y}_{1}, x_{1}\right)\right)=0$, thus, Case (a) is not true. Similarly, Case (b) is not true, too.

Case (c). When $u_{1} \in T\left(x_{1}, y_{1}\right), v_{1} \in T\left(y_{1}, x_{1}\right)$, as proved above, we can get that

$$
\begin{aligned}
& \mathrm{D}\left(\mathrm{x}_{1}, \mathrm{~T}\left(\mathrm{x}_{1}, \mathrm{y}_{1}\right)\right)=\mathrm{D}\left(\mathrm{u}_{1}, \mathrm{~T}\left(\mathrm{x}_{1}, \mathrm{y}_{1}\right)\right)=\operatorname{dist}(A, \mathrm{~B})=0, \\
& \mathrm{D}\left(\mathrm{y}_{1}, \mathrm{~T}\left(\mathrm{x}_{1}, \mathrm{y}_{1}\right)\right)=\mathrm{D}\left(v_{1}, \mathrm{~T}\left(\mathrm{y}_{1}, x_{1}\right)\right)=\operatorname{dist}(A, \mathrm{~B})=0,
\end{aligned}
$$

which imply that $\left(x_{1}, y_{1}\right)$ is the coupled best proximity point. So we only consider Case (d), that is, $u_{1} \notin T\left(x_{1}, y_{1}\right), v_{1} \notin T\left(y_{1}, x_{1}\right)$. Because $T\left(x_{1}, y_{1}\right)$ and $T\left(y_{1}, x_{1}\right)$ are closed in $B$, therefore

$$
\mathrm{D}\left(\mathrm{u}_{1}, \mathrm{~T}\left(\mathrm{x}_{1}, \mathrm{y}_{1}\right)\right)>0, \mathrm{D}\left(v_{1}, \mathrm{~T}\left(\mathrm{y}_{1}, \mathrm{x}_{1}\right)\right)>0 \text {. }
$$

Since $u_{1} \in T\left(x_{0}, y_{0}\right), v_{1} \in T\left(y_{0}, x_{0}\right)$, it is obvious that

$$
0<D\left(u_{1}, T\left(x_{1}, y_{1}\right)\right) \leqslant H\left(T\left(x_{0}, y_{0}\right), T\left(x_{1}, y_{1}\right)\right),
$$

and

$$
0<\mathrm{D}\left(v_{1}, \mathrm{~T}\left(\mathrm{y}_{1}, \mathrm{x}_{1}\right)\right) \leqslant \mathrm{H}\left(\mathrm{T}\left(\mathrm{y}_{0}, \mathrm{x}_{0}\right), \mathrm{T}\left(\mathrm{y}_{1}, \mathrm{x}_{1}\right)\right) .
$$

Applying Lemma 3.5, we obtain that for $q_{0}>1, q_{0}^{\prime}>1$, there exist $u_{2} \in T\left(x_{1}, y_{1}\right), v_{2} \in T\left(y_{1}, x_{1}\right)$ such that

$$
0<\mathrm{d}\left(\mathrm{u}_{1}, \mathrm{u}_{2}\right)<\mathrm{q}_{0} \mathrm{D}\left(\mathrm{u}_{1}, \mathrm{~T}\left(\mathrm{x}_{1}, \mathrm{y}_{1}\right)\right), 0<\mathrm{d}\left(v_{1}, v_{2}\right)<\mathrm{q}_{0}^{\prime} \mathrm{D}\left(v_{1}, \mathrm{~T}\left(\mathrm{y}_{1}, \mathrm{x}_{1}\right)\right) .
$$

On the other hand, as $u_{2} \in T\left(x_{1}, y_{1}\right) \subseteq B_{0}, v_{2} \in T\left(y_{1}, x_{1}\right) \subseteq B_{0}$, there exist $x_{2} \neq x_{1}, y_{2} \neq y_{1} \in A_{0}$, for otherwise $\left(x_{1}, y_{1}\right)$ is the coupled best proximity point, such that

$$
\mathrm{d}\left(\mathrm{x}_{2}, \mathrm{u}_{2}\right)=\mathrm{d}\left(\mathrm{y}_{2}, v_{2}\right)=\operatorname{dist}(\mathrm{A}, \mathrm{B}) .
$$

Since $T$ is an $\alpha^{+}$-proximal admissible, $u_{2} \in T\left(x_{1}, y_{1}\right), v_{2} \in T\left(y_{1}, x_{1}\right)$ and $\alpha\left(x_{0}, x_{1}\right) \geqslant 0, \alpha\left(y_{0}, y_{1}\right) \geqslant 0$, using (3.2), we obtain $\alpha\left(x_{1}, x_{2}\right) \geqslant 0$ and $\alpha\left(y_{1}, y_{2}\right) \geqslant 0$, that is,

$$
\begin{aligned}
& d\left(x_{2}, u_{2}\right)=\operatorname{dist}(A, B), \quad \alpha\left(x_{1}, x_{2}\right) \geqslant 0, \\
& d\left(y_{2}, v_{2}\right)=\operatorname{dist}(A, B), \quad \alpha\left(y_{1}, y_{2}\right) \geqslant 0 .
\end{aligned}
$$

Because (A, B) satisfies the weak P-property and in combination with (3.2), (3.6), we have

$$
d\left(x_{1}, x_{2}\right) \leqslant d\left(u_{1}, u_{2}\right), d\left(y_{1}, y_{2}\right) \leqslant d\left(v_{1}, v_{2}\right) .
$$

From (3.3), (3.4), (3.5) and (3.8), we derive

$$
\begin{aligned}
& d\left(x_{1}, x_{2}\right) \leqslant d\left(u_{1}, u_{2}\right)<q_{0} D\left(u_{1}, T\left(x_{1}, y_{1}\right)\right) \leqslant q_{0} H\left(T\left(x_{0}, y_{0}\right), T\left(x_{1}, y_{1}\right)\right), \\
& d\left(y_{1}, y_{2}\right) \leqslant d\left(v_{1}, v_{2}\right)<q_{0}^{\prime} D\left(v_{1}, T\left(y_{1}, x_{1}\right)\right) \leqslant q_{0}^{\prime} H\left(T\left(y_{0}, x_{0}\right), T\left(y_{1}, x_{1}\right)\right) .
\end{aligned}
$$

Likewise, assuming that $u_{2} \notin T\left(x_{2}, y_{2}\right), v_{2} \notin T\left(y_{2}, x_{2}\right)$; for otherwise, condition (iv) is not true or $\left(x_{2}, y_{2}\right)$ 
is the coupled best proximity point. Because $T\left(x_{2}, y_{2}\right)$ and $T\left(y_{2}, x_{2}\right)$ are closed in $B$, therefore

$$
\mathrm{D}\left(\mathrm{u}_{2}, \mathrm{~T}\left(\mathrm{x}_{2}, \mathrm{y}_{2}\right)\right)>0, \quad \mathrm{D}\left(v_{2}, \mathrm{~T}\left(\mathrm{y}_{2}, v_{2}\right)\right)>0 .
$$

Thus, by $u_{2} \in\left(x_{1}, y_{1}\right), v_{2} \in\left(y_{1}, x_{1}\right)$, we have

$$
\begin{aligned}
& 0<\mathrm{D}\left(\mathrm{u}_{2}, \mathrm{~T}\left(\mathrm{x}_{2}, \mathrm{y}_{2}\right)\right) \leqslant \mathrm{H}\left(\mathrm{T}\left(\mathrm{x}_{1}, \mathrm{y}_{1}\right), \mathrm{T}\left(\mathrm{x}_{2}, \mathrm{y}_{2}\right)\right), \\
& 0<\mathrm{D}\left(v_{2}, \mathrm{~T}\left(\mathrm{y}_{2}, \mathrm{x}_{2}\right)\right) \leqslant \mathrm{H}\left(\mathrm{T}\left(\mathrm{y}_{1}, \mathrm{x}_{1}\right), \mathrm{T}\left(\mathrm{y}_{2}, \mathrm{x}_{2}\right)\right) .
\end{aligned}
$$

Applying Lemma 3.5, we obtain that for $q_{1}>1, q_{1}^{\prime}>1$, there exist $u_{3} \in T\left(x_{2}, y_{2}\right), v_{3} \in T\left(y_{2}, x_{2}\right)$ such that

$$
0<\mathrm{d}\left(\mathrm{u}_{2}, \mathrm{u}_{3}\right)<\mathrm{q}_{1} \mathrm{D}\left(\mathrm{u}_{2}, \mathrm{~T}\left(\mathrm{x}_{2}, \mathrm{y}_{2}\right)\right), \quad 0<\mathrm{d}\left(v_{2}, v_{3}\right)<\mathrm{q}_{1}^{\prime} \mathrm{D}\left(v_{2}, \mathrm{~T}\left(\mathrm{y}_{2}, \mathrm{x}_{2}\right)\right) \text {. }
$$

On the other hand, as $u_{3} \in T\left(x_{2}, y_{2}\right) \subseteq B_{0}, v_{3} \in T\left(y_{2}, x_{2}\right) \subseteq B_{0}$, there exist $x_{3} \neq x_{2}, y_{3} \neq y_{2} \in A_{0}$, for otherwise $\left(x_{2}, y_{2}\right)$ is the coupled best proximity point, such that

$$
\mathrm{d}\left(\mathrm{x}_{3}, \mathrm{u}_{3}\right)=\mathrm{d}\left(\mathrm{y}_{3}, v_{3}\right)=\operatorname{dist}(\mathrm{A}, \mathrm{B}) .
$$

Again, since $T$ is an $\alpha^{+}$-proximal admissible, $u_{3} \in T\left(x_{2}, y_{2}\right), v_{3} \in T\left(y_{2}, x_{2}\right)$ and $\alpha\left(x_{1}, x_{2}\right) \geqslant 0, \alpha\left(y_{1}, y_{2}\right) \geqslant 0$, we obtain $\alpha\left(x_{2}, x_{3}\right) \geqslant 0$ and $\alpha\left(y_{2}, y_{3}\right) \geqslant 0$, that is,

$$
\begin{aligned}
& d\left(x_{3}, u_{3}\right)=\operatorname{dist}(A, B), \quad \alpha\left(x_{2}, x_{3}\right) \geqslant 0, \\
& d\left(y_{3}, v_{3}\right)=\operatorname{dist}(A, B), \quad \alpha\left(y_{2}, y_{3}\right) \geqslant 0 .
\end{aligned}
$$

Because $(A, B)$ satisfies the weak P-property and in combination with (3.7), (3.11), we have

$$
d\left(x_{2}, x_{3}\right) \leqslant d\left(u_{2}, u_{3}\right), \quad d\left(y_{2}, y_{3}\right) \leqslant d\left(v_{2}, v_{3}\right) .
$$

From (3.9), (3.10) and (3.12), we have

$$
\begin{aligned}
& d\left(x_{2}, x_{3}\right) \leqslant d\left(u_{2}, u_{3}\right)<q_{1} D\left(u_{2}, T\left(x_{2}, y_{2}\right)\right) \leqslant q_{1} H\left(T\left(x_{1}, y_{1}\right), T\left(x_{2}, y_{2}\right)\right), \\
& d\left(y_{2}, y_{3}\right) \leqslant d\left(v_{2}, v_{3}\right)<q_{1}^{\prime} D\left(v_{2}, T\left(y_{2}, x_{2}\right)\right) \leqslant q_{1}^{\prime} H\left(T\left(y_{1}, x_{1}\right), T\left(y_{2}, x_{2}\right)\right),
\end{aligned}
$$

for all $n \in \mathbb{N} \backslash\{0\}$. Inductively, we can obtain sequences $\left\{x_{n}\right\}_{\mathfrak{n}=0}^{\infty},\left\{y_{n}\right\}_{n=0}^{\infty} \subseteq A_{0}$ and $\left\{u_{n}\right\}_{\mathfrak{n}=0}^{\infty},\left\{v_{n}\right\}_{n=0}^{\infty} \subseteq B_{0}$ satisfying $x_{n} \neq x_{n+1}, y_{n} \neq y_{n+1}, D\left(u_{n}, T\left(x_{n}, y_{n}\right)\right)>0, D\left(v_{n}, T\left(y_{n}, x_{n}\right)\right)>0, u_{n+1} \in T\left(x_{n}, y_{n}\right)$ and $v_{n+1} \in T\left(y_{n}, x_{n}\right), n \in \mathbb{N}$ such that

$$
\begin{gathered}
\mathrm{d}\left(x_{n+1}, u_{n+1}\right)=\operatorname{dist}(A, B), \quad \alpha\left(x_{n}, x_{n+1}\right) \geqslant 0, \quad \forall n \in \mathbb{N}, \\
d\left(y_{n+1}, v_{n+1}\right)=\operatorname{dist}(A, B), \quad \alpha\left(y_{n}, y_{n+1}\right) \geqslant 0, \quad \forall n \in \mathbb{N}, \\
d\left(x_{n}, x_{n+1}\right) \leqslant d\left(u_{n}, u_{n+1}\right), \quad \forall n \in \mathbb{N}, \\
d\left(y_{n}, y_{n+1}\right) \leqslant d\left(v_{n}, v_{n+1}\right), \quad \forall n \in \mathbb{N},
\end{gathered}
$$

and

$$
\begin{aligned}
& d\left(x_{n}, x_{n+1}\right) \leqslant d\left(u_{n}, u_{n+1}\right)<q_{n-1} D\left(u_{n}, T\left(x_{n}, y_{n}\right)\right) \leqslant q_{n-1} H\left(T\left(x_{n-1}, y_{n-1}\right), T\left(x_{n}, y_{n}\right)\right), \\
& d\left(y_{n}, y_{n+1}\right) \leqslant d\left(v_{n}, v_{n+1}\right)<q_{n-1}^{\prime} D\left(v_{n}, T\left(y_{n}, x_{n}\right)\right) \leqslant q_{n-1}^{\prime} H\left(T\left(y_{n-1}, x_{n-1}\right), T\left(y_{n}, x_{n}\right)\right),
\end{aligned}
$$

for all $n \in \mathbb{N} \backslash\{0\}$. Since $x_{n} \in A_{0}, T\left(x_{n-1}, y_{n-1}\right) \subseteq B_{0}$ and $A_{0} \subseteq A, B_{0} \subseteq B$, from the definition of $D$ and (3.13), we have

$$
\begin{aligned}
& \operatorname{dist}(A, B)=d\left(x_{n}, u_{n}\right) \geqslant D\left(x_{n}, T\left(x_{n-1}, y_{n-1}\right)\right) \geqslant \operatorname{dist}\left(A_{0}, B_{0}\right) \geqslant \operatorname{dist}(A, B), \\
& \operatorname{dist}(A, B)=d\left(y_{n}, v_{n}\right) \geqslant D\left(y_{n}, T\left(y_{n-1}, x_{n-1}\right)\right) \geqslant \operatorname{dist}\left(A_{0}, B_{0}\right) \geqslant \operatorname{dist}(A, B),
\end{aligned}
$$

for all $n \in \mathbb{N} \backslash\{0\}$, hence,

$$
\begin{aligned}
& d\left(x_{n}, u_{n}\right)=D\left(x_{n}, T\left(x_{n-1}, y_{n-1}\right)\right)=\operatorname{dist}(A, B), \alpha\left(x_{n}, x_{n+1}\right) \geqslant 0 \\
& d\left(y_{n}, v_{n}\right)=D\left(y_{n}, T\left(y_{n-1}, x_{n-1}\right)\right)=\operatorname{dist}(A, B), \alpha\left(y_{n}, y_{n+1}\right) \geqslant 0 .
\end{aligned}
$$


In addition, we deduce that

$$
\begin{aligned}
\frac{1}{2} D^{*}\left(x_{n-1}, T\left(x_{n-1}, y_{n-1}\right)\right) & =\frac{1}{2}\left[D\left(x_{n-1}, T\left(x_{n-1}, y_{n-1}\right)\right)-\operatorname{dist}(A, B)\right] \\
& \leqslant \frac{1}{2}\left[d\left(x_{n-1}, x_{n}\right)+D\left(x_{n}, T\left(x_{n-1}, y_{n-1}\right)\right)-\operatorname{dist}(A, B)\right] \\
& =\frac{1}{2} d\left(x_{n-1}, x_{n}\right) \\
& \leqslant d\left(x_{n-1}, x_{n}\right),
\end{aligned}
$$

and

$$
\begin{aligned}
\frac{1}{2} D^{*}\left(y_{n-1}, T\left(y_{n-1}, x_{n-1}\right)\right) & =\frac{1}{2}\left[D\left(y_{n-1}, T\left(y_{n-1}, x_{n-1}\right)\right)-\operatorname{dist}(A, B)\right] \\
& \leqslant \frac{1}{2}\left[d\left(y_{n-1}, y_{n}\right)+D\left(y_{n}, T\left(y_{n-1}, x_{n-1}\right)\right)-\operatorname{dist}(A, B)\right] \\
& =\frac{1}{2} d\left(y_{n-1}, y_{n}\right) \\
& \leqslant d\left(y_{n-1}, y_{n}\right),
\end{aligned}
$$

for all $n \in \mathbb{N} \backslash\{0\}$. Thus, by (3.1), we get

$$
\begin{aligned}
\theta\left(q H\left(T\left(x_{n-1}, y_{n-1}\right), T\left(x_{n}, y_{n}\right)\right)\right) & \leqslant \alpha\left(x_{n-1}, x_{n}\right)+\theta\left(q H\left(T\left(x_{n-1}, y_{n-1}\right), T\left(x_{n}, y_{n}\right)\right)\right) \\
& \leqslant\left[\theta\left(M\left(x_{n-1}, x_{n}, y_{n-1}, y_{n}\right)\right)\right]^{k} \\
\theta\left(q H\left(T\left(y_{n-1}, x_{n-1}\right), T\left(y_{n}, x_{n}\right)\right)\right) & \leqslant \alpha\left(y_{n-1}, y_{n}\right)+H\left(q T\left(y_{n-1}, x_{n-1}\right), T\left(y_{n}, x_{n}\right)\right) \\
& \leqslant\left[\theta\left(M\left(y_{n-1}, y_{n}, x_{n-1}, x_{n}\right)\right)\right]^{k} .
\end{aligned}
$$

We check

$$
\begin{aligned}
& M\left(x_{n-1}, x_{n}, y_{n-1}, y_{n}\right) \\
&= \max \left\{d\left(x_{n-1}, x_{n}\right), \frac{D\left(x_{n-1}, T\left(x_{n-1}, y_{n-1}\right)\right)+D\left(x_{n}, T\left(x_{n}, y_{n}\right)\right)}{2}\right. \\
&\left.-\operatorname{dist}(A, B), \frac{D\left(x_{n}, T\left(x_{n-1}, y_{n-1}\right)\right)+D\left(x_{n-1}, T\left(x_{n}, y_{n}\right)\right)}{2}-\operatorname{dist}(A, B)\right\} \\
& \leqslant \max \left\{d\left(x_{n-1}, x_{n}\right), \frac{1}{2}\left[d\left(x_{n-1}, x_{n}\right)+D\left(x_{n}, T\left(x_{n-1}, y_{n-1}\right)\right)\right]\right. \\
&+\frac{1}{2}\left[d\left(x_{n}, x_{n+1}\right)+D\left(x_{n+1}, T\left(x_{n}, y_{n}\right)\right)\right]-\operatorname{dist}(A, B) \\
&\left.\frac{1}{2}\left[\operatorname{dist}(A, B)+d\left(x_{n-1}, x_{n}\right)+d\left(x_{n}, x_{n+1}\right)+D\left(x_{n+1}, T\left(x_{n}, y_{n}\right)\right)\right]-\operatorname{dist}(A, B)\right\} \\
& \leqslant \max \left\{d\left(x_{n-1}, x_{n}\right), \frac{1}{2}\left[d\left(x_{n-1}, x_{n}\right)+d\left(x_{n}, x_{n+1}\right)\right], \frac{1}{2}\left[d\left(x_{n-1}, x_{n}\right)+d\left(x_{n}, x_{n+1}\right)\right]\right\} \\
& \leqslant \max \left\{d\left(x_{n-1}, x_{n}\right), d\left(x_{n}, x_{n+1}\right)\right\},
\end{aligned}
$$

for all $n \in \mathbb{N} \backslash\{0\}$. Similarly, we get

$$
M\left(y_{n-1}, y_{n}, x_{n-1}, x_{n}\right) \leqslant \max \left\{d\left(y_{n-1}, y_{n}\right), d\left(y_{n}, y_{n+1}\right)\right\}
$$

Set $q_{n-1}=q_{n-1}^{\prime}=q$ for every $n \in \mathbb{N} \backslash\{0\}$. From (3.14), (3.15), (3.16), (3.17) and (3.18), we get

$$
\begin{aligned}
\theta\left(d\left(x_{n}, x_{n+1}\right)\right) & \leqslant \theta\left(d\left(u_{n}, u_{n+1}\right)\right) \\
& \leqslant \theta\left(q H\left(T\left(x_{n-1}, y_{n-1}\right), T\left(x_{n}, y_{n}\right)\right)\right) \\
& \leqslant\left[\theta\left(M\left(x_{n-1}, x_{n}, y_{n-1}, y_{n}\right)\right)\right]^{k} \\
& =\left[\theta\left(\max \left\{d\left(x_{n-1}, x_{n}\right), d\left(x_{n}, x_{n+1}\right)\right\}\right)\right]^{k},
\end{aligned}
$$


and

$$
\begin{aligned}
\theta\left(d\left(y_{n}, y_{n+1}\right)\right) & \leqslant \theta\left(d\left(v_{n}, v_{n+1}\right)\right) \\
& \leqslant \theta\left(q H\left(T\left(y_{n-1}, x_{n-1}\right), T\left(y_{n}, x_{n}\right)\right)\right) \\
& \leqslant\left[\theta\left(M\left(y_{n-1}, y_{n}, x_{n-1}, x_{n}\right)\right)\right]^{k} \\
& =\left[\theta\left(\max \left\{d\left(y_{n-1}, y_{n}\right), d\left(y_{n}, y_{n+1}\right)\right\}\right)\right]^{k}
\end{aligned}
$$

If we assume that

$$
\begin{aligned}
& \max \left\{d\left(x_{n-1}, x_{n}\right), d\left(x_{n}, x_{n+1}\right)\right\}=d\left(x_{n}, x_{n+1}\right), \\
& \max \left\{d\left(y_{n-1}, y_{n}\right), d\left(y_{n}, y_{n+1}\right)\right\}=d\left(y_{n}, y_{n+1}\right),
\end{aligned}
$$

then we get the contradictive inequalities

$$
\begin{aligned}
\theta\left(d\left(x_{n}, x_{n+1}\right)\right) & \leqslant\left[\theta\left(M\left(x_{n-1}, x_{n}, y_{n-1}, y_{n}\right)\right)\right]^{k} \\
& =\left[\theta\left(d\left(x_{n}, x_{n+1}\right)\right)\right]^{k} \\
& <\theta\left(d\left(x_{n}, x_{n+1}\right)\right)
\end{aligned}
$$

and

$$
\begin{aligned}
\theta\left(d\left(y_{n}, y_{n+1}\right)\right) & \leqslant\left[\theta\left(M\left(y_{n-1}, y_{n}, x_{n-1}, x_{n}\right)\right)\right]^{k} \\
& =\left[\theta\left(d\left(y_{n}, y_{n+1}\right)\right)\right]^{k} \\
& <\theta\left(d\left(y_{n}, y_{n+1}\right)\right)
\end{aligned}
$$

thus,

$$
\begin{aligned}
& \max \left\{d\left(x_{n-1}, x_{n}\right), d\left(x_{n}, x_{n+1}\right)\right\}=d\left(x_{n-1}, x_{n}\right), \\
& \max \left\{d\left(y_{n-1}, y_{n}\right), d\left(y_{n}, y_{n+1}\right)\right\}=d\left(y_{n-1}, y_{n}\right),
\end{aligned}
$$

hence,

$$
\theta\left(d\left(x_{n}, x_{n+1}\right)\right) \leqslant \theta\left(d\left(u_{n}, u_{n+1}\right)\right) \leqslant\left[\theta\left(d\left(x_{n-1}, x_{n}\right)\right)\right]^{k},
$$

and

$$
\theta\left(d\left(y_{n}, y_{n+1}\right)\right) \leqslant \theta\left(d\left(v_{n}, v_{n+1}\right)\right) \leqslant\left[\theta\left(d\left(y_{n-1}, y_{n}\right)\right)\right]^{k},
$$

for all $n \in \mathbb{N} \backslash\{0\}$. Therefore, we obtain

$$
\begin{aligned}
1 & \leqslant \theta\left(d\left(x_{n}, x_{n+1}\right)\right) \leqslant\left[\theta\left(d\left(x_{n-1}, x_{n}\right)\right)\right]^{k} \\
& \leqslant\left[\theta\left(d\left(x_{n-2}, x_{n-1}\right)\right)\right]^{k^{2}} \leqslant \ldots \leqslant\left[\theta\left(d\left(x_{0}, x_{1}\right)\right)\right]^{k^{n}}, \\
1 & \leqslant \theta\left(d\left(y_{n}, y_{n+1}\right)\right) \leqslant\left[\theta\left(d\left(y_{n-1}, y_{n}\right)\right)\right]^{k} \\
& \leqslant\left[\theta\left(d\left(y_{n-2}, y_{n-1}\right)\right)\right]^{k^{2}} \leqslant \ldots \leqslant\left[\theta\left(d\left(y_{0}, y_{1}\right)\right)\right]^{k^{n}}, \\
1 & \leqslant \theta\left(d\left(u_{n}, u_{n+1}\right)\right) \leqslant\left[\theta\left(d\left(x_{n-1}, x_{n}\right)\right)\right]^{k} \\
& \leqslant\left[\theta\left(d\left(x_{n-2}, x_{n-1}\right)\right)\right]^{k^{2}} \leqslant \ldots \leqslant\left[\theta\left(d\left(x_{0}, x_{1}\right)\right)\right]^{k^{n}},
\end{aligned}
$$

and

$$
\begin{aligned}
1 & \leqslant \theta\left(q d\left(v_{n}, v_{n+1}\right)\right) \leqslant\left[\theta\left(d\left(y_{n-1}, y_{n}\right)\right)\right]^{k} \\
& \leqslant\left[\theta\left(d\left(y_{n-2}, y_{n-1}\right)\right)\right]^{k^{2}} \leqslant \ldots \leqslant\left[\theta\left(d\left(y_{0}, y_{1}\right)\right)\right]^{k^{n}} .
\end{aligned}
$$

Letting $n \rightarrow \infty$ in (3.20), (3.21), (3.22), and (3.23), we get

$$
\begin{aligned}
& \theta\left(d\left(x_{n}, x_{n+1}\right)\right) \rightarrow 1, \quad \theta\left(d\left(y_{n}, y_{n+1}\right)\right) \rightarrow 1, \\
& \theta\left(d\left(u_{n}, u_{n+1}\right)\right) \rightarrow 1, \quad \theta\left(d\left(v_{n}, v_{n+1}\right)\right) \rightarrow 1,
\end{aligned}
$$


as $n \rightarrow \infty$. According to the properties of function $\theta$, there exist $0<r<1$ and $0<\ell \leqslant \infty$ such that

$$
\begin{aligned}
& \lim _{n \rightarrow \infty} \frac{\theta\left(d\left(x_{n}, x_{n+1}\right)\right)-1}{\left[d\left(x_{n}, x_{n+1}\right)\right]^{r}}=\lim _{n \rightarrow \infty} \frac{\theta\left(d\left(y_{n}, y_{n+1}\right)\right)-1}{\left[d\left(y_{n}, y_{n+1}\right)\right]^{r}}=\ell, \\
& \lim _{n \rightarrow \infty} \frac{\theta\left(d\left(u_{n}, u_{n+1}\right)\right)-1}{\left[d\left(u_{n}, u_{n+1}\right)\right]^{r}}=\lim _{n \rightarrow \infty} \frac{\theta\left(d\left(v_{n}, v_{n+1}\right)\right)-1}{\left[d\left(v_{n}, v_{n+1}\right)\right]^{r}}=\ell,
\end{aligned}
$$

when $\ell<\infty$, taking $M=\frac{\ell}{2}>0$ and using the definition of the limit, there exists $n_{0} \in \mathbb{N} \backslash\{0\}$ such that

$$
\begin{aligned}
& \left|\frac{\theta\left(d\left(x_{n}, x_{n+1}\right)\right)-1}{\left[d\left(x_{n}, x_{n+1}\right)\right]^{r}}-\ell\right| \leqslant M, \quad\left|\frac{\theta\left(d\left(y_{n}, y_{n+1}\right)\right)-1}{\left[d\left(y_{n}, y_{n+1}\right)\right]^{r}}-\ell\right| \leqslant M, \\
& \left|\frac{\theta\left(d\left(u_{n}, u_{n+1}\right)\right)-1}{\left[d\left(u_{n}, u_{n+1}\right)\right]^{r}}-\ell\right| \leqslant M, \quad\left|\frac{\theta\left(d\left(v_{n}, v_{n+1}\right)\right)-1}{\left[d\left(v_{n}, v_{n+1}\right)\right]^{r}}-\ell\right| \leqslant M,
\end{aligned}
$$

for all $n \geqslant n_{0}$. Thus

$$
\frac{\theta\left(d\left(x_{n}, x_{n+1}\right)\right)-1}{\left[d\left(x_{n}, x_{n+1}\right)\right]^{r}} \geqslant M, \quad \frac{\theta\left(d\left(y_{n}, y_{n+1}\right)\right)-1}{\left[d\left(y_{n}, y_{n+1}\right)\right]^{r}} \geqslant M
$$

and

$$
\frac{\theta\left(d\left(u_{n}, u_{n+1}\right)\right)-1}{\left[d\left(u_{n}, u_{n+1}\right)\right]^{r}} \geqslant M, \quad \frac{\theta\left(d\left(v_{n}, v_{n+1}\right)\right)-1}{\left[d\left(v_{n}, v_{n+1}\right)\right]^{r}} \geqslant M
$$

for all $n \geqslant n_{0}$. Subsequently,

$$
\begin{aligned}
& n\left[d\left(x_{n}, x_{n+1}\right)\right]^{r} \leqslant n M^{\prime}\left[\theta\left(d\left(x_{n}, x_{n+1}\right)\right)-1\right], \\
& n\left[d\left(y_{n}, y_{n+1}\right)\right]^{r} \leqslant n M^{\prime}\left[\theta\left(d\left(y_{n}, y_{n+1}\right)\right)-1\right], \\
& n\left[d\left(u_{n}, u_{n+1}\right)\right]^{r} \leqslant n M^{\prime}\left[\theta\left(d\left(u_{n}, u_{n+1}\right)\right)-1\right],
\end{aligned}
$$

and

$$
n\left[d\left(v_{n}, v_{n+1}\right)\right]^{r} \leqslant n M^{\prime}\left[\theta\left(d\left(v_{n}, v_{n+1}\right)\right)-1\right],
$$

for all $n \geqslant n_{0}$, where $M^{\prime}=\frac{1}{M}$. Suppose now that $\ell=\infty$. Let $M>0$ be an arbitrary positive number. From the definition of the limit, there exists $n \in \mathbb{N}$ such that

$$
\frac{\theta\left(d\left(x_{n}, x_{n+1}\right)\right)-1}{\left[d\left(x_{n}, x_{n+1}\right)\right]^{r}} \geqslant M, \quad \frac{\theta\left(d\left(y_{n}, y_{n+1}\right)\right)-1}{\left[d\left(y_{n}, y_{n+1}\right)\right]^{r}} \geqslant M
$$

and

$$
\frac{\theta\left(d\left(u_{n}, u_{n+1}\right)\right)-1}{\left[d\left(u_{n}, u_{n+1}\right)\right]^{r}} \geqslant M, \quad \frac{\theta\left(d\left(v_{n}, v_{n+1}\right)\right)-1}{\left[d\left(v_{n}, v_{n+1}\right)\right]^{r}} \geqslant M
$$

for all $n \geqslant n_{0}$. This implies

$$
\begin{aligned}
& n\left[d\left(x_{n}, x_{n+1}\right)\right]^{r} \leqslant n M^{\prime}\left[\theta\left(d\left(x_{n}, x_{n+1}\right)\right)-1\right], \\
& n\left[d\left(y_{n}, y_{n+1}\right)\right]^{r} \leqslant n M^{\prime}\left[\theta\left(d\left(y_{n}, y_{n+1}\right)\right)-1\right], \\
& n\left[d\left(u_{n}, u_{n+1}\right)\right]^{r} \leqslant n M^{\prime}\left[\theta\left(d\left(u_{n}, u_{n+1}\right)\right)-1\right],
\end{aligned}
$$

and

$$
\mathrm{n}\left[\mathrm{d}\left(v_{\mathrm{n}}, v_{\mathrm{n}+1}\right)\right]^{\mathrm{r}} \leqslant \mathrm{n} \mathrm{M}^{\prime}\left[\theta\left(\mathrm{d}\left(v_{\mathrm{n}}, v_{\mathrm{n}+1}\right)\right)-1\right],
$$

for all $n \geqslant n_{0}$, where $M^{\prime}=\frac{1}{M}$. Thus either of $\ell=\infty$ and $\ell<\infty$, the inequalities

$$
\begin{aligned}
& n\left[d\left(x_{n}, x_{n+1}\right)\right]^{r} \leqslant n M^{\prime}\left[\theta\left(d\left(x_{n}, x_{n+1}\right)\right)-1\right], \\
& n\left[d\left(y_{n}, y_{n+1}\right)\right]^{r} \leqslant n M^{\prime}\left[\theta\left(d\left(y_{n}, y_{n+1}\right)\right)-1\right],
\end{aligned}
$$




$$
n\left[d\left(u_{n}, u_{n+1}\right)\right]^{r} \leqslant n M^{\prime}\left[\theta\left(d\left(u_{n}, u_{n+1}\right)\right)-1\right]
$$

and

$$
\mathrm{n}\left[\mathrm{d}\left(v_{\mathrm{n}}, v_{\mathrm{n}+1}\right)\right]^{\mathrm{r}} \leqslant \mathrm{n} \mathrm{M}^{\prime}\left[\theta\left(\mathrm{d}\left(v_{\mathrm{n}}, v_{\mathrm{n}+1}\right)\right)-1\right],
$$

are true for all $n \geqslant n_{0}$. Applying (3.20), (3.21), (3.22), (3.23) to above inequalities, we get

$$
\begin{aligned}
& n\left[d\left(x_{n}, x_{n+1}\right)\right]^{r} \leqslant n M^{\prime}\left(\left[\theta\left(d\left(x_{n}, x_{n+1}\right)\right)\right]^{k^{n}}-1\right), \\
& n\left[d\left(y_{n}, y_{n+1}\right)\right]^{r} \leqslant n M^{\prime}\left(\left[\theta\left(d\left(y_{n}, y_{n+1}\right)\right)\right]^{k^{n}}-1\right), \\
& n\left[d\left(u_{n}, u_{n+1}\right)\right]^{r} \leqslant n M^{\prime}\left(\left[\theta\left(d\left(u_{n}, u_{n+1}\right)\right)\right]^{k^{n}}-1\right),
\end{aligned}
$$

and

$$
\mathrm{n}\left[\mathrm{d}\left(v_{\mathrm{n}}, v_{\mathrm{n}+1}\right)\right]^{\mathrm{r}} \leqslant \mathrm{n} \mathrm{M}^{\prime}\left(\left[\theta\left(\mathrm{d}\left(v_{\mathrm{n}}, v_{\mathrm{n}+1}\right)\right)\right]^{\mathrm{k}^{\mathrm{n}}}-1\right),
$$

for all $n \geqslant n_{0}$. Letting $n \rightarrow \infty$ in the above inequality, we obtain

$$
\begin{array}{ll}
\lim _{n \rightarrow \infty} n\left[d\left(x_{n}, x_{n+1}\right)\right]^{r}=0, & \lim _{n \rightarrow \infty} n\left[d\left(y_{n}, y_{n+1}\right)\right]^{r}=0, \\
\lim _{n \rightarrow \infty} n\left[d\left(u_{n}, u_{n+1}\right)\right]^{r}=0, & \lim _{n \rightarrow \infty} n\left[d\left(v_{n}, v_{n+1}\right)\right]^{r}=0 .
\end{array}
$$

Subsequently, there exists $n_{1} \in \mathbb{N}$ such that when $n \geqslant n_{1}$,

$$
\begin{array}{ll}
n\left[d\left(x_{n}, x_{n+1}\right)\right]^{r} \leqslant 1, & n\left[d\left(y_{n}, y_{n+1}\right)\right]^{r} \leqslant 1, \\
n\left[d\left(u_{n}, u_{n+1}\right)\right]^{r} \leqslant 1, & n\left[d\left(v_{n}, v_{n+1}\right)\right]^{r} \leqslant 1 .
\end{array}
$$

Hence, when $n \geqslant n_{1}$,

$$
\begin{aligned}
& d\left(x_{n}, x_{n+1}\right) \leqslant \frac{1}{n^{1 / r}}, \quad d\left(y_{n}, y_{n+1}\right) \leqslant \frac{1}{n^{1 / r}}, \\
& d\left(u_{n}, u_{n+1}\right) \leqslant \frac{1}{n^{1 / r}}, \quad d\left(v_{n}, v_{n+1}\right) \leqslant \frac{1}{n^{1 / r}} .
\end{aligned}
$$

Letting $n>m>n_{1}$ and applying the triangle inequality repeatedly, we get

$$
\begin{aligned}
& d\left(x_{m}, x_{n}\right) \leqslant \sum_{i=m}^{n-1} d\left(x_{i}, x_{i+1}\right) \leqslant \sum_{i=m}^{n-1} \frac{1}{i^{1 / r}}, \\
& d\left(x_{m}, x_{n}\right) \leqslant \sum_{i=m}^{n-1} d\left(y_{i}, y_{i+1}\right) \leqslant \sum_{i=m}^{n-1} \frac{1}{i^{1 / r}}, \\
& d\left(x_{m}, x_{n}\right) \leqslant \sum_{i=m}^{n-1} d\left(u_{i}, u_{i+1}\right) \leqslant \sum_{i=m}^{n-1} \frac{1}{i^{1 / r}},
\end{aligned}
$$

and

$$
d\left(x_{m}, x_{n}\right) \leqslant \sum_{i=m}^{n-1} d\left(v_{i}, v_{i+1}\right) \leqslant \sum_{i=m}^{n-1} \frac{1}{i^{1 / r}} .
$$

Because $0<r<1$, therefore $\sum_{i=m}^{\infty} \frac{1}{\mathfrak{i}^{1 / r}}$ is convergent. Thus letting $m, n \rightarrow \infty$ in (3.24), (3.25), (3.26), (3.27), we deduce that $\left\{x_{\mathfrak{n}}\right\}_{\mathfrak{n}=0}^{\infty},\left\{y_{\mathfrak{n}}\right\}_{\mathfrak{n}=0}^{\infty},\left\{u_{\mathfrak{n}}\right\}_{\mathfrak{n}=0}^{\infty}$ and $\left\{v_{\mathfrak{n}}\right\}_{\mathfrak{n}=0}^{\infty}$ are Cauchy sequence in $(X, d)$. Since $A$ and $B$ are closed subsets of complete metric space $(X, d)$, thus, there exists $\left(x^{*}, y^{*}\right) \in A \times A$ such that

$$
x_{k} \stackrel{d}{\rightarrow} x^{*} \text { as } k \rightarrow \infty, \text { and } y_{k} \stackrel{d}{\rightarrow} y^{*} \text { as } k \rightarrow \infty,
$$

likewise, there exists $\left(u^{*}, v^{*}\right) \in A \times A$ such that

$$
\mathrm{u}_{\mathrm{k}} \stackrel{\mathrm{d}}{\rightarrow} \mathrm{u}^{*} \text { as } \mathrm{k} \rightarrow \infty, \text { and } v_{\mathrm{k}} \stackrel{\mathrm{d}}{\rightarrow} v^{*} \text { as } \mathrm{k} \rightarrow \infty .
$$

If $(v)$ holds, then from (3.15), noting that $u_{n} \in T\left(x_{n-1}, x_{n}\right)$ and $v_{n} \in T\left(y_{n-1}, y_{n}\right)$, it is easy to derive that 


$$
\begin{aligned}
& d\left(x^{*}, u^{*}\right)=D\left(x^{*}, T\left(x^{*}, y^{*}\right)\right)=\operatorname{dist}(A, B), \\
& d\left(y^{*}, v^{*}\right)=D\left(y^{*}, T\left(y^{*}, x^{*}\right)\right)=\operatorname{dist}(A, B) .
\end{aligned}
$$

If (vi) holds, then $\alpha\left(x_{k}, x^{*}\right) \geqslant 0$. We can conclude that

$$
\begin{aligned}
& \frac{1}{2} D^{*}\left(x_{n}, T\left(x_{n}, y_{n}\right)\right) \leqslant d\left(x_{n}, x^{*}\right), \\
& \frac{1}{2} D^{*}\left(y_{n}, T\left(y_{n}, x_{n}\right)\right) \leqslant d\left(y_{n}, y^{*}\right),
\end{aligned}
$$

or

$$
\begin{aligned}
& \frac{1}{2} D^{*}\left(x_{n+1}, T\left(x_{n+1}, y_{n+1}\right)\right) \leqslant d\left(x_{n+1}, x^{*}\right), \\
& \frac{1}{2} D^{*}\left(y_{n+1}, T\left(y_{n+1}, x_{n+1}\right)\right) \leqslant d\left(y_{n+1}, y^{*}\right),
\end{aligned}
$$

hold for all $n \in \mathbb{N}$. In fact, assume that

$$
\frac{1}{2} D^{*}\left(x_{n}, T\left(x_{n}, y_{n}\right)\right)>d\left(x_{n}, x^{*}\right), \frac{1}{2} D^{*}\left(y_{n}, T\left(y_{n}, x_{n}\right)\right)>d\left(y_{n}, y^{*}\right),
$$

and

$$
\frac{1}{2} D^{*}\left(x_{n+1}, T\left(x_{n+1}, y_{n+1}\right)\right)>d\left(x_{n+1}, x^{*}\right), \frac{1}{2} D^{*}\left(y_{n+1}, T\left(y_{n+1}, x_{n+1}\right)\right)>d\left(y_{n+1}, y^{*}\right),
$$

are true for some $n \in \mathbb{N}$. Since $x_{n} \neq x_{n+1}$ and $y_{n} \neq y_{n+1}$, for all $n \in \mathbb{N}$, then $d\left(x_{n}, x_{n+1}\right)>0$ and $d\left(y_{n}, y_{n+1}\right)>0$, for all $n \in \mathbb{N}$. Moreover, using (3.15), (3.19), we derive the following contradictive inequalities

$$
\begin{aligned}
d\left(x_{n}, x_{n+1}\right) \leqslant & d\left(x_{n}, x^{*}\right)+d\left(x_{n+1}, x^{*}\right) \\
< & \frac{1}{2}\left[D^{*}\left(x_{n}, T\left(x_{n}, y_{n}\right)\right)+D^{*}\left(x_{n+1}, T\left(x_{n+1}, y_{n+1}\right)\right)\right] \\
= & \frac{1}{2}\left[D\left(x_{n}, T\left(x_{n}, y_{n}\right)\right)+D\left(x_{n+1}, T\left(x_{n+1}, y_{n+1}\right)\right)-2 \operatorname{dist}(A, B)\right] \\
\leqslant & \frac{1}{2}\left[d\left(x_{n}, x_{n+1}\right)+D\left(x_{n+1}, T\left(x_{n}, y_{n}\right)\right)+d\left(x_{n+1}, x_{n+2}\right)\right. \\
& \left.+D\left(x_{n+2}, T\left(x_{n+1}, y_{n+1}\right)\right)-2 \operatorname{dist}(A, B)\right] \\
= & \frac{1}{2}\left[d\left(x_{n}, x_{n+1}\right)+d\left(x_{n+1}, x_{n+2}\right)\right] \\
< & d\left(x_{n}, x_{n+1}\right), \\
d\left(y_{n}, y_{n+1}\right) \leqslant & d\left(y_{n}, y^{*}\right)+d\left(y_{n+1}, x^{*}\right) \\
< & \frac{1}{2}\left[D *\left(y_{n}, T\left(y_{n}, x_{n}\right)\right)+D^{*}\left(y_{n+1}, T\left(y_{n+1}, x_{n+1}\right)\right)\right] \\
= & \frac{1}{2}\left[D\left(y_{n}, T\left(y_{n}, x_{n}\right)\right)+D\left(y_{n+1}, T\left(y_{n+1}, x_{n+1}\right)\right)-2 \operatorname{dist}(A, B)\right] \\
= & \frac{1}{2}\left[d\left(y_{n}, y_{n+1}\right)+D\left(y_{n+1}, T\left(y_{n}, x_{n}\right)\right)+d\left(y_{n+1}, y_{n+2}\right)\right. \\
& \left.+D\left(y_{n+2}, T\left(y_{n+1}, x_{n+1}\right)\right)-2 d i s t(A, B)\right] \\
\leqslant & \frac{1}{2}\left[d\left(y_{n}, y_{n+1}\right)+d\left(y_{n+1}, y_{n+2}\right)\right] \\
< & d\left(y_{n}, y_{n+1}\right),
\end{aligned}
$$

hence, either (3.28) or (3.29) holds, notice that $\left\{x_{n+1}\right\}_{n=0}^{\infty}$ is a subsequence of $\left\{x_{n}\right\}_{n=0}^{\infty}$, consequently, we can verify that there exists at least a subsequence $\left\{x_{n_{k}}\right\}$ of $\left\{x_{n}\right\}_{\mathfrak{n}=0}^{\infty}$ such that for all $k \in \mathbb{N}$,

$$
\frac{1}{2} D^{*}\left(x_{n_{k}}, T\left(x_{n_{k}}, y_{n_{k}}\right)\right) \leqslant d\left(x_{n_{k}}, x^{*}\right), \frac{1}{2} D^{*}\left(y_{n_{k}}, T\left(y_{n_{k}}, x_{n_{k}}\right)\right) \leqslant d\left(y_{n_{k}}, y^{*}\right),
$$


and $\alpha\left(x_{n_{k}}, x^{*}\right) \geqslant 0, \alpha\left(y_{n_{k}}, y^{*}\right) \geqslant 0$ hold. From (3.1), we obtain

$$
\begin{aligned}
\theta\left(q H\left(T\left(x_{n_{k}}, y_{n_{k}}\right), T\left(x^{*}, y^{*}\right)\right)\right) & \leqslant\left[\theta\left(M\left(x_{n_{k}}, x^{*}, y_{n_{k}}, y^{*}\right)\right)\right]^{k} \\
& <\theta\left(M\left(x_{n_{k}}, x^{*}, y_{n_{k}}, y^{*}\right)\right), \\
\theta\left(q H\left(T\left(y_{n_{k}}, x_{n_{k}}\right), T\left(y^{*}, x^{*}\right)\right)\right) & \leqslant\left[\theta\left(M\left(y_{n_{k}}, y^{*}, x_{n_{k}}, x^{*}\right)\right)\right]^{k} \\
& <\theta\left(M\left(y_{n_{k}}, y^{*}, x_{n_{k}}, x^{*}\right)\right),
\end{aligned}
$$

which implies

$$
\begin{aligned}
& 0<q H\left(T\left(x_{n_{k}}, y_{n_{k}}\right), T\left(x^{*}, y^{*}\right)\right)<M\left(x_{n_{k}}, x^{*}, y_{n_{k}}, y^{*}\right), \\
& 0<q H\left(T\left(y_{n_{k}}, x_{n_{k}}\right), T\left(y^{*}, x^{*}\right)\right)<M\left(y_{n_{k}}, y^{*}, x_{n_{k}}, x^{*}\right),
\end{aligned}
$$

hence,

$$
\begin{aligned}
& 0<H\left(T\left(x_{n_{k}}, y_{n_{k}}\right), T\left(x^{*}, y^{*}\right)\right)<M\left(x_{n_{k}}, x^{*}, y_{n_{k}}, y^{*}\right), \\
& 0<H\left(T\left(y_{n_{k}}, x_{n_{k}}\right), T\left(y^{*}, x^{*}\right)\right)<M\left(y_{n_{k}}, y^{*}, x_{n_{k}}, x^{*}\right) .
\end{aligned}
$$

Subsequently,

$$
\begin{aligned}
& \lim _{k \rightarrow \infty} H\left(T\left(x_{n_{k}}, y_{n_{k}}\right), T\left(x^{*}, y^{*}\right)\right) \leqslant \lim _{k \rightarrow \infty} M\left(x_{n_{k}}, x^{*}, y_{n_{k}}, y^{*}\right), \\
& \lim _{k \rightarrow \infty} H\left(T\left(y_{n_{k}}, x_{n_{k}}\right), T\left(y^{*}, x^{*}\right)\right) \leqslant \lim _{k \rightarrow \infty} M\left(y_{n_{k}}, y^{*}, x_{n_{k}}, x^{*}\right) .
\end{aligned}
$$

\section{Moreover,}

$$
\begin{aligned}
M\left(x_{n_{k}}, x^{*}, y_{n_{k}}, y^{*}\right)= & \max \left\{d\left(x_{n_{k}}, x^{*}\right), \frac{D\left(x_{n_{k}}, T\left(x_{n_{k}}, y_{n_{k}}\right)\right)+D\left(x^{*}, T\left(x^{*}, y^{*}\right)\right)}{2}\right. \\
& \left.-\operatorname{dist}(A, B), \frac{D\left(x^{*}, T\left(x_{n_{k}}, y_{n_{k}}\right)\right)+D\left(x_{n_{k}}, T\left(x^{*}, y^{*}\right)\right)}{2}-\operatorname{dist}(A, B)\right\} \\
\leqslant & \max \left\{d\left(x_{n_{k}}, x^{*}\right), \frac{d\left(x_{n_{k}}, x_{n_{k}+1}\right)+D\left(x_{n_{k}+1}, T\left(x_{n_{k}}, y_{n_{k}}\right)\right)+D\left(x^{*}, T\left(x^{*}, y^{*}\right)\right)}{2}\right. \\
& -\operatorname{dist}(A, B), \frac{d\left(x^{*}, x_{n_{k}+1}\right)+D\left(x_{n_{k}+1}, T\left(x_{n_{k}}, y_{n_{k}}\right)\right)}{2} \\
& \left.+\frac{D\left(x_{n_{k}}, x^{*}\right)+D\left(x^{*}, T\left(x^{*}, y^{*}\right)\right)}{2}-\operatorname{dist}(A, B)\right\} \\
\leqslant & \max \left\{d\left(x_{n_{k}}, x^{*}\right), \frac{d\left(x_{n_{k}}, x_{n_{k}+1}\right)+\operatorname{dist}(A, B)+D\left(x^{*}, T\left(x^{*}, y^{*}\right)\right)}{2}-\operatorname{dist}(A, B),\right. \\
& \left.\frac{d\left(x^{*}, x_{n_{k}+1}\right)+\operatorname{dist}(A, B)+D\left(x_{n_{k}}, x^{*}\right)+D\left(x^{*}, T\left(x^{*}, y^{*}\right)\right)}{2}-\operatorname{dist}(A, B)\right\},
\end{aligned}
$$

which implies that

$$
\lim _{k \rightarrow \infty} M\left(x_{n_{k}}, x^{*}, y_{n_{k}}, y^{*}\right) \leqslant \frac{D\left(x^{*}, T\left(x^{*}, y^{*}\right)\right)-\operatorname{dist}(A, B)}{2} .
$$

Similarly, we get

$$
\lim _{k \rightarrow \infty} M\left(y_{n_{k}}, y^{*}, x_{n_{k}}, x^{*}\right) \leqslant \frac{D\left(y^{*}, T\left(y^{*}, x^{*}\right)\right)-\operatorname{dist}(A, B)}{2} .
$$

Additionally, we have

$$
\begin{aligned}
D\left(x^{*}, T\left(x^{*}, y^{*}\right)\right) & \leqslant d\left(x^{*}, x_{n_{k}}\right)+d\left(x_{n_{k}}, u_{n_{k}}\right)+D\left(u_{n_{k}}, T\left(x^{*}, y^{*}\right)\right) \\
& \leqslant d\left(x^{*}, x_{n_{k}}\right)+d\left(x_{n_{k}}, u_{n_{k}}\right)+H\left(T\left(x_{n_{k}}, y_{n_{k}}\right), T\left(x^{*}, y^{*}\right)\right) \\
& \leqslant d\left(x^{*}, x_{n_{k}}\right)+\operatorname{dist}(A, B)+H\left(T\left(x_{n_{k}}, y_{n_{k}}\right), T\left(x^{*}, y^{*}\right)\right),
\end{aligned}
$$

and

$$
\begin{aligned}
D\left(y^{*}, T\left(y^{*}, x^{*}\right)\right) & \leqslant d\left(y^{*}, y_{n_{k}}\right)+d\left(y_{n_{k}}, v_{n_{k}}\right)+D\left(v_{n_{k}}, T\left(y^{*}, x^{*}\right)\right) \\
& \leqslant d\left(y^{*}, y_{n_{k}}\right)+d\left(y_{n_{k}}, v_{n_{k}}\right)+H\left(T\left(y_{n_{k}}, x_{n_{k}}\right), T\left(y^{*}, x^{*}\right)\right) \\
& \leqslant d\left(y^{*}, y_{n_{k}}\right)+\operatorname{dist}(A, B)+H\left(T\left(y_{n_{k}}, x_{n_{k}}\right), T\left(y^{*}, x^{*}\right)\right),
\end{aligned}
$$


which implies

$$
D\left(x^{*}, T\left(x^{*}, y^{*}\right)\right)-\operatorname{dist}(A, B) \leqslant d\left(x^{*}, x_{n_{k}}\right)+H\left(T\left(x_{n_{k}}, y_{n_{k}}\right), T\left(x^{*}, y^{*}\right)\right)
$$

and

$$
D\left(y^{*}, T\left(y^{*}, x^{*}\right)\right)-\operatorname{dist}(A, B) \leqslant d\left(y^{*}, y_{n_{k}}\right)+H\left(T\left(y_{n_{k}}, x_{n_{k}}\right), T\left(y^{*}, x^{*}\right)\right) .
$$

Letting $k \rightarrow \infty$ in, we derive

$$
\begin{aligned}
& D\left(x^{*}, T\left(x^{*}, y^{*}\right)\right)-\operatorname{dist}(A, B) \leqslant \lim _{k \rightarrow \infty} H\left(T\left(x_{n_{k}}, y_{n_{k}}\right), T\left(x^{*}, y^{*}\right)\right), \\
& D\left(y^{*}, T\left(y^{*}, x^{*}\right)\right)-\operatorname{dist}(A, B) \leqslant \lim _{k \rightarrow \infty} H\left(T\left(y_{n_{k}}, x_{n_{k}}\right), T\left(y^{*}, x^{*}\right)\right) .
\end{aligned}
$$

Therefore, the inequalities

$$
D\left(x^{*}, T\left(x^{*}, y^{*}\right)\right)-\operatorname{dist}(A, B) \leqslant \frac{D\left(x^{*}, T\left(x^{*}, y^{*}\right)\right)-\operatorname{dist}(A, B)}{2},
$$

and

$$
\mathrm{D}\left(\mathrm{y}^{*}, \mathrm{~T}\left(\mathrm{y}^{*}, x^{*}\right)\right)-\operatorname{dist}(\mathrm{A}, \mathrm{B}) \leqslant \frac{\mathrm{D}\left(\mathrm{y}^{*}, \mathrm{~T}\left(\mathrm{y}^{*}, x^{*}\right)\right)-\operatorname{dist}(\mathrm{A}, \mathrm{B})}{2},
$$

hold, which implies that

$$
D\left(x^{*}, T\left(x^{*}, y^{*}\right)\right)=\operatorname{dist}(A, B), D\left(y^{*}, T\left(y^{*}, x^{*}\right)\right)=\operatorname{dist}(A, B),
$$

that is, $\operatorname{CBpp}(\mathrm{T})$ is nonempty.

The following example is provided to support the results of Theorem 3.8, we take the same $\theta$ function as that in example of [7].

Example 3.9. Let $X=R^{2}$ be equipped with the metric

$$
\mathrm{d}(\mathrm{P}, \mathrm{Q})=\sqrt{|\mathrm{p}-\mathrm{q}|^{2}+\left|\mathrm{p}^{\prime}-\mathrm{q}^{\prime}\right|^{2}}
$$

for any $P=\left(p, p^{\prime}\right), Q=\left(q, q^{\prime}\right) \in X$. Let $A=A_{1} \cup A_{2} \cup A_{3}, B=\left\{P=\left(p, p^{\prime}\right) \in X: p=2,0 \leqslant p^{\prime} \leqslant 1\right\}$, where

$$
\begin{aligned}
& A_{1}=\left\{P=\left(p, p^{\prime}\right) \in X: p=0,0 \leqslant p^{\prime} \leqslant 1\right\}, \\
& A_{2}=\left\{P=\left(p, p^{\prime}\right) \in X: p=1,0 \leqslant p^{\prime} \leqslant 1\right\}, \\
& A_{3}=\left\{P=\left(p, p^{\prime}\right) \in X: p=\frac{1}{2}, p^{\prime}=0\right\} .
\end{aligned}
$$

Obviously, $\operatorname{dist}(A, B)=1, A_{0}=\{(0,1),(1,1)\}, B_{0}=\{(0,2),(1,2)\}$. Let

$$
\begin{aligned}
& B_{1}=\left\{P=\left(p, p^{\prime}\right) \in X: 0 \leqslant p=2,0 \leqslant p^{\prime} \leqslant \frac{1}{2}\right\}, \\
& B_{2}=\left\{P=\left(p, p^{\prime}\right) \in X: 0 \leqslant p=2, \frac{1}{2} \leqslant p^{\prime} \leqslant 1\right\} .
\end{aligned}
$$

Define the mapping $T: A \times A \rightarrow C L(B)$, for $P, Q \in A$,

$$
T(P, Q)= \begin{cases}\{(0,2)\}, & \text { if } P=(0,1), \\ \{(1,2)\}, & \text { if } P=(1,1), \\ \left\{\left(\frac{1}{2}, 2\right)\right\}, & \text { if } P=\left(\frac{1}{2}, 0\right), \\ B_{1}, & \text { if } P \in A_{1} \backslash\{(0,1)\}, \\ B_{2}, & \text { if } P \in A_{2} \backslash\{(1,1)\} .\end{cases}
$$

Obviously, $T\left(A_{0} \times A_{0}\right) \subseteq B_{0}$. Let $P_{1}=\left(p_{1}, p_{1}^{\prime}\right), P_{2}=\left(p_{2}, p_{2}^{\prime}\right) \in A_{0}, Q_{1}=\left(q_{1}, q_{1}^{\prime}\right), Q_{2}=\left(q_{2}, q_{2}^{\prime}\right) \in B_{0}$. We obtain that

$$
d\left(P_{1}, P_{2}\right)=1=d\left(Q_{1}, Q_{1}\right)
$$

Consequently, $d\left(P_{1}, P_{2}\right) \leqslant d\left(Q_{1}, Q_{2}\right)$, for $P_{1}, P_{2} \in A_{0}, Q_{1}, Q_{2} \in B_{0}$. So $(A, B)$ satisfy the weak P-property. 
Now, consider functions $\theta:(0,+\infty) \rightarrow(1,+\infty)$ by [7]

$$
\theta(\mathrm{t}):=e^{\sqrt{t e^{t}}} .
$$

It is not difficult to show $\theta \in \Theta$. Define function $\alpha: A \times A \rightarrow[-\infty,+\infty)$

$$
\alpha(x, y):= \begin{cases}0, & x, y \in\left\{(0,1),(1,1),\left(\frac{1}{2}, 0\right)\right\}, \\ -\infty, & \text { otherwise. }\end{cases}
$$

Suppose that for any $x_{1}, x_{2}, w_{1}, w_{2}, w_{1}^{\prime}, w_{2}^{\prime}, y_{1}, y_{2} \in A$ and $u_{1} \in T\left(x_{1}, y_{1}\right), u_{2} \in T\left(x_{2}, y_{2}\right), v_{1} \in T\left(y_{1}, x_{1}\right), v_{2} \in$ $\mathrm{T}\left(\mathrm{y}_{2}, \mathrm{x}_{2}\right)$,

$$
\left\{\begin{array}{l}
\alpha\left(x_{1}, x_{2}\right) \geqslant 0, \\
d\left(w_{1}, u_{1}\right)=\operatorname{dist}(A, B), \\
d\left(w_{2}, u_{2}\right)=\operatorname{dist}(A, B),
\end{array}\right.
$$

and

$$
\left\{\begin{array}{l}
\alpha\left(y_{1}, y_{2}\right) \geqslant 0, \\
d\left(w_{1}^{\prime}, v_{1}\right)=\operatorname{dist}(A, B), \\
d\left(w_{2}^{\prime}, v_{2}\right)=\operatorname{dist}(A, B),
\end{array}\right.
$$

we deduce by the definition of $\alpha$ that $x_{i}, y_{i} \in\left\{(0,1),(1,1),\left(\frac{1}{2}, 0\right)\right\}$ for $i=1,2$. Moreover, $d\left(w_{i}, u_{i}\right)=$ $\operatorname{dist}(A, B)$, if and only if $w_{i}=(0,1), u_{i}=(0,2)$ or $w_{i}=(1,1), u_{i}=(1,2)$ for $i=1,2$. It follows that $\alpha\left(w_{1}, w_{2}\right) \geqslant 0$. Similarly, $\alpha\left(w_{1}^{\prime}, w_{2}^{\prime}\right) \geqslant 0$. Thus, $T$ is a proximal $\alpha^{+}$-admissible mapping.

Assume that $\mathrm{H}(\mathrm{T}(\mathrm{P}, \mathrm{Q}), \mathrm{T}(\mathrm{U}, \mathrm{V}))>0, \mathrm{H}(\mathrm{T}(\mathrm{U}, \mathrm{V}), \mathrm{T}(\mathrm{P}, \mathrm{Q}))>0$, and

$$
\left.\begin{array}{l}
\frac{1}{2} D^{*}(P, T(P, Q)) \leqslant d(P, U), \\
\frac{1}{2} D^{*}(Q, T(Q, P)) \leqslant d(Q, V), \\
d(P, U)=d(Q, V)=0, \text { or } \\
d(P, U)>0, d(Q, V)>0,
\end{array}\right\} \quad \text { and } \quad \alpha(P, U) \geqslant 0, \alpha(Q, V) \geqslant 0 \text {, }
$$

for $P, Q, U, V \in A$. When $P=Q=U=V=(1,1)$ or $P=Q=U=V=(0,1)$,

$$
D^{*}(P, T(P, Q))=D^{*}(Q, T(Q, P))=d(P, U)=d(Q, V)=0,
$$

in this case, it is clear that both $((1,1),(1,1))$ and $((0,1),(0,1))$ are the coupled best proximity point. The remainder cases satisfying (3.13) are as follows:

$$
\begin{aligned}
& \text { (i). }\left\{\begin{array} { l } 
{ P \in \{ ( \frac { 1 } { 2 } , 0 ) \} , U \in \{ ( 0 , 1 ) \} , } \\
{ Q \in \{ ( \frac { 1 } { 2 } , 0 ) \} , V \in \{ ( 1 , 1 ) \} , }
\end{array} \text { or } \quad \left\{\begin{array}{l}
P \in\left\{\left(\frac{1}{2}, 0\right)\right\}, U \in\{(1,1)\}, \\
Q \in\left\{\left(\frac{1}{2}, 0\right)\right\}, V \in\{(0,1)\},
\end{array}\right.\right. \\
& \text { (ii). }\left\{\begin{array}{l}
P \in\left\{\left(\frac{1}{2}, 0\right)\right\}, U \in\{(0,1),(1,1)\}, \\
Q \in\{(0,1)\}, V \in\left\{(1,1),\left(\frac{1}{2}, 0\right)\right\},
\end{array}\right. \\
& \text { (iii). }\left\{\begin{array} { l } 
{ P \in \{ ( \frac { 1 } { 2 } , 0 ) \} , U \in \{ ( 0 , 1 ) \} , } \\
{ Q \in \{ ( 1 , 1 ) \} , \quad V \in \{ ( \frac { 1 } { 2 } , 0 ) \} , }
\end{array} \text { or } \quad \left\{\begin{array}{l}
P \in\left\{\left(\frac{1}{2}, 0\right)\right\}, U \in\{(1,1)\}, \\
Q \in\{(1,1)\}, V \in\{(0,1)\},
\end{array}\right.\right. \\
& \text { (iv). }\left\{\begin{array}{l}
P \in\{(0,1)\}, U \in\left\{(1,1),\left(\frac{1}{2}, 0\right)\right\}, \\
Q \in\left\{\left(\frac{1}{2}, 0\right)\right\}, V \in\{(0,1),(1,1)\},
\end{array}\right. \\
& \text { (v). }\left\{\begin{array}{l}
P \in\{(0,1)\}, U \in\left\{(1,1),\left(\frac{1}{2}, 0\right)\right\}, \\
Q \in\{(1,1)\}, V \in\left\{(0,1),\left(\frac{1}{2}, 0\right)\right\},
\end{array}\right. \\
& \text { (vi). }\left\{\begin{array} { l } 
{ P \in \{ ( 1 , 1 ) \} , U \in \{ ( \frac { 1 } { 2 } , 0 ) \} , } \\
{ Q \in \{ ( 1 , 1 ) \} , V \in \{ ( 0 , 1 ) \} , }
\end{array} \text { or } \quad \left\{\begin{array}{l}
P \in\{(1,1)\}, U \in\{(0,1)\}, \\
Q \in\{(1,1)\}, V \in\left\{\left(\frac{1}{2}, 0\right)\right\},
\end{array}\right.\right. \\
& \text { (vii). }\left\{\begin{array}{l}
P \in\{(1,1)\}, U \in\left\{\left(\frac{1}{2}, 0\right),(0,1)\right\}, \\
Q \in\{(0,1)\}, V \in\left\{\left(\frac{1}{2}, 0\right),(1,1)\right\},
\end{array}\right. \\
& \text { (viii). }\left\{\begin{array} { l } 
{ P \in \{ ( 1 , 1 ) \} , U \in \{ ( \frac { 1 } { 2 } , 0 ) \} , } \\
{ Q \in \{ ( \frac { 1 } { 2 } , 0 ) \} , V \in \{ ( 0 , 1 ) \} , }
\end{array} \text { or } \quad \left\{\begin{array}{l}
P \in\{(1,1)\}, U \in\{(0,1)\}, \\
Q \in\left\{\left(\frac{1}{2}, 0\right)\right\}, V \in\{(1,1)\} .
\end{array}\right.\right.
\end{aligned}
$$


We discuss these eight cases:

Case (i). $d(P, U)=d(Q, V)=\frac{\sqrt{5}}{2}$.

Case (ii). $d(P, U)=\frac{\sqrt{5}}{2}$ and $d(Q, V)=\frac{\sqrt{5}}{2}$ or $d(Q, V)=1$.

Case (iii). $d(P, U)=\frac{\sqrt{5}}{2}$ and $d(Q, V)=1$ or $d(Q, V)=\frac{\sqrt{5}}{2}$.

Case (iv). $d(P, U)=\frac{\sqrt{5}}{2}$ or $d(P, U)=1$ and $d(Q, V)=\frac{\sqrt{5}}{2}$.

Case $(v) \cdot d(P, U)=\frac{\sqrt{5}}{2}$ or $d(P, U)=1$ and $d(Q, V)=\frac{\sqrt{5}}{2}$ or $d(Q, V)=1$.

Case $(v i) \cdot d(P, U)=1$ or $d(P, U)=\frac{\sqrt{5}}{2}$ and $d(Q, V)=\frac{\sqrt{5}}{2}$ or $d(Q, V)=1$.

Case (vii). $d(P, U)=1$ or $d(P, U)=\frac{\sqrt{5}}{2}$ and $d(Q, V)=1$ or $d(Q, V)=\frac{\sqrt{5}}{2}$.

Case (viii). $d(P, U)=1$ or $d(P, U)=\frac{\sqrt{5}}{2}$ and $d(Q, V)=\frac{\sqrt{5}}{2}$.

For all cases (i)-(viii), we obtain that $H(T(P, Q), T(U, V))=H(T(U, V), T(P, Q))=\frac{1}{2}$ and $\min \{d(P, U), d(Q, V)\}=1$. Calculating

$$
\begin{aligned}
& \theta\left(\frac{10}{9} H(T(P, Q), T(U, V))\right)=\theta\left(\frac{5}{9}\right)=e^{\sqrt{\frac{5}{9}} e^{\frac{5}{9}}} \\
& {[\theta(\min \{d(P, U), d(Q, V)\})]^{8 / 9}=(\theta(1))^{8 / 9}=\left(e^{\sqrt{e}}\right)^{8 / 9}}
\end{aligned}
$$

since

$$
\begin{aligned}
e^{\sqrt{\frac{5}{9} e^{\frac{5}{9}}}} \leqslant\left(e^{\sqrt{e}}\right)^{8 / 9} & \Leftarrow \sqrt{\frac{5}{9} e^{\frac{5}{9}}} \leqslant \frac{8 \sqrt{e}}{9} \\
& \Leftarrow \frac{5}{9} e^{\frac{5}{9}} \leqslant \frac{64 e}{81} \\
& \Leftarrow \frac{45}{64} e^{\frac{5}{9}} \leqslant e \\
& \Leftarrow \frac{45}{64}<1<e^{\frac{4}{9}}
\end{aligned}
$$

hence,

$$
\theta\left(\frac{10}{9} H(T(P, Q), T(U, V))\right) \leqslant[\theta(\min \{d(P, U), d(Q, V)\})]^{8 / 9} \leqslant[\theta(M(P, Q, U, V))]^{8 / 9}
$$

and

$$
\theta\left(\frac{10}{9} \mathrm{H}(\mathrm{T}(\mathrm{U}, \mathrm{V}), \mathrm{T}(\mathrm{P}, \mathrm{Q}))\right) \leqslant[\theta(\min \{\mathrm{d}(\mathrm{P}, \mathrm{U}), \mathrm{d}(\mathrm{Q}, \mathrm{V})\})]^{8 / 9} \leqslant[\theta(\mathrm{M}(\mathrm{U}, \mathrm{V}, \mathrm{P}, \mathrm{Q}))]^{8 / 9} .
$$

Comprehensively, for all cases (i)-(viii), we obtain that when $\mathrm{H}(\mathrm{T}(\mathrm{P}, \mathrm{Q}), \mathrm{T}(\mathrm{U}, \mathrm{V}))>0, \mathrm{H}(\mathrm{T}(\mathrm{U}, \mathrm{V}), \mathrm{T}(\mathrm{P}, \mathrm{Q}))>$ $0, \alpha(P, U) \geqslant 0, \alpha(Q, V) \geqslant 0$, and $q=\frac{10}{9}, k=8 / 9$,

$$
\left.\begin{array}{l}
\frac{1}{2} D^{*}(P, T(P, Q)) \leqslant d(P, U), \\
\frac{1}{2} D^{*}(Q, T(Q, P)) \leqslant d(Q, V), \\
d(P, U)=d(Q, V)=0, \text { or } \\
d(P, U)>0, d(Q, V)>0,
\end{array}\right\} \Rightarrow \alpha(P, U)+\theta(q H(T(P, Q), T(U, V))) \leqslant[\theta(M(P, Q, U, V))]^{k} .
$$


In addition, $\mathrm{D}(\mathrm{P}, \mathrm{T}(\mathrm{P}, \mathrm{Q})) \neq 0$ for all cases, hence, the condition (iv) holds in Theorem 3.8. Thus all the assumptions of Theorem 3.8 are satisfied and there exists $((0,1),(1,1)) \in A_{0} \times A_{0}$ such that

$$
\begin{aligned}
& \mathrm{D}((0,1), \mathrm{T}((0,1),(1,1)))=1=\operatorname{dist}(\mathrm{A}, \mathrm{B}), \\
& \mathrm{D}((1,1), \mathrm{T}((1,1),(0,1)))=1=\operatorname{dist}(A, B),
\end{aligned}
$$

that is, $\operatorname{CBpp}(T)=\{((0,1),(1,1)),((1,1),(1,1)),((0,1),(0,1))\}$.

If we replace the condition that $\mathrm{T}$ is a two-variable Suzuki type $\alpha^{+}-\theta$-proximal multivalued mapping with

$$
\alpha(x, y)+\theta\left(q H\left(T\left(x, x^{\prime}\right), T\left(y, y^{\prime}\right)\right)\right) \leqslant\left[\theta\left(M\left(x, y, x^{\prime}, y^{\prime}\right)\right)\right]^{k},
$$

then the next result can be deduced easily from Theorem 3.8.

Corollary 3.10. Let $\mathrm{X}, \mathrm{A}, \mathrm{A}_{0}$, and $\mathrm{B}$ be as in Theorem 3.8. Assume that $\mathrm{T}: \mathrm{A} \times \mathrm{A} \rightarrow \operatorname{CL}(\mathrm{B})$ satisfies the assertions (i)-(vi) in Theorem 3.8 and

$$
\alpha(x, y)+\theta\left(q H\left(T\left(x, x^{\prime}\right), T\left(y, y^{\prime}\right)\right)\right) \leqslant\left[\theta\left(M\left(x, y, x^{\prime}, y^{\prime}\right)\right)\right]^{k},
$$

holds for all $x, y, x^{\prime}, y^{\prime} \in A$, where $\alpha: A \times A \rightarrow[-\infty,+\infty), q>1$, and $\theta \in \Theta$. Then CBpp $(T)$ is nonempty.

Especially, letting $\alpha(x, y)=0$ for all $x, y \in A$ in Theorem 3.8, obviously, (ii), (iii), and (vi) hold in Theorem 3.8, thus we state the following new result.

Corollary 3.11. Suppose that $X, A, A_{0}$, and $B$ are as defined in Theorem 3.8 and $\theta \in \Theta . T: A \times A \rightarrow \operatorname{CL}(B)$ satisfies the following assertions:

(i) $\mathrm{T}(\mathrm{x}, \mathrm{y}) \subseteq \mathrm{B}_{0}$ for $(\mathrm{x}, \mathrm{y}) \in \mathrm{A}_{0} \times \mathrm{A}_{0}$ and $(\mathrm{A}, \mathrm{B})$ satisfies the weak P-property;

(ii) there exist $\mathrm{q}>1$ and $0<\mathrm{k}<1$ such that

$$
\left.\begin{array}{l}
\frac{1}{2} D^{*}\left(x, T\left(x, x^{\prime}\right)\right) \leqslant d(x, y), \\
\frac{1}{2} D^{*}\left(x^{\prime}, T\left(x^{\prime}, x\right)\right) \leqslant d\left(x^{\prime}, y^{\prime}\right), \\
d(x, y)=d\left(x^{\prime}, y^{\prime}\right)=0, \text { or } \\
d(x, y)>0, d\left(x^{\prime}, y^{\prime}\right)>0,
\end{array}\right\} \Rightarrow \theta\left(q H\left(T\left(x, x^{\prime}\right), T\left(y, y^{\prime}\right)\right)\right) \leqslant\left[\theta\left(M\left(x, y, x^{\prime}, y^{\prime}\right)\right)\right]^{k},
$$

holds for all $x, y, x^{\prime}, y^{\prime} \in A$;

(iii) if $\mathrm{D}(x, \mathrm{~T}(x, y))=0$ or $\mathrm{D}(\mathrm{y}, \mathrm{T}(\mathrm{y}, \mathrm{x}))=0$ for any $\mathrm{x}, \mathrm{y} \in \mathrm{A}_{0}$, then $\mathrm{D}(\mathrm{x}, \mathrm{T}(\mathrm{x}, \mathrm{y}))=\mathrm{D}(\mathrm{y}, \mathrm{T}(\mathrm{y}, \mathrm{x}))=0$.

Then $\mathrm{CBpp}(\mathrm{T})$ is nonempty.

If we reduce the two variables of the mapping $T: A \times A \rightarrow C L(B)$ to one-variable in Theorem 3.8, Corollary 3.10, and Corollary 3.11, respectively, applying Definitions 3.2, 3.6 to $T$ and simplifying proof processes of Theorem 3.8, Corollary 3.10, and Corollary 3.11 to one-variable version of $T$, it is easy to see that these results are still true, thus, we state the following results with the omitting proofs.

Theorem 3.12. Let $(X, d)$ be a complete metric space and $A, B \in \mathcal{C L}(X)$ with $A_{0} \neq \emptyset . \theta \in \Theta$ and $T: A \rightarrow \operatorname{CL}(B)$ be a one-variable Suzuki type $\alpha^{+}-\theta$-proximal multivalued mapping. Suppose that the following conditions hold:

(i) $\mathrm{T} x \subseteq \mathrm{B}_{0}$ for $\mathrm{x} \in \mathrm{A}_{0}$ and $(\mathrm{A}, \mathrm{B})$ satisfies the weak P-property;

(ii) $\mathrm{T}$ is $\alpha^{+}$-proximal admissible; 
(iii) there exist elements $x_{0}, x_{1} \in A_{0}$, and $u_{1} \in T x_{0}$ such that

$$
d\left(x_{1}, u_{1}\right)=\operatorname{dist}(A, B), \quad \alpha\left(x_{0}, x_{1}\right) \geqslant 0 ;
$$

(iv) T is continuous; or

(v) if $\left\{x_{n}\right\}_{\mathfrak{n}=0}^{\infty}$ is a sequence in $A$ such that $\alpha\left(x_{n}, x_{n+1}\right) \geqslant 0$ and $x_{n} \rightarrow x^{*} \in A$ as $n \rightarrow \infty$, then $\alpha\left(x_{n}, x^{*}\right) \geqslant 0$ for all $\mathrm{n} \in \mathbb{N}$.

Then $\operatorname{Bpp}(\mathrm{T})$ is nonempty.

Corollary 3.13. Let $\mathrm{X}, \mathrm{A}, \mathrm{A}_{0}$, and $\mathrm{B}$ be as in Theorem 3.12. Assume that $\mathrm{T}: \mathrm{A} \rightarrow \mathrm{CL}(\mathrm{B})$ satisfies the assertions (i)-(v) in Theorem 3.12 and

$$
\alpha(x, y)+\theta(q H(T x, T y)) \leqslant[\theta(M(x, y))]^{k},
$$

holds for all $x, y \in A$, where $\alpha: A \times A \rightarrow[-\infty,+\infty)$ and $q>1$. Then $\operatorname{Bpp}(T)$ is nonempty.

Corollary 3.14. Suppose that $X, A, A_{0}$, and $B$ are as defined in Theorem 3.12 and $\theta \in \Theta . T: A \rightarrow \operatorname{CL}(B)$ satisfies the following assertions:

(i) $\mathrm{T} x \subseteq \mathrm{B}_{0}$ for $\mathrm{x} \in \mathrm{A}_{0}$ and $(\mathrm{A}, \mathrm{B})$ satisfies the weak P-property;

(ii)

$$
\frac{1}{2} D^{*}(x, T x) \leqslant d(x, y) \Rightarrow \theta(q H(T x, T y)) \leqslant\left[\theta(M(x, y)]^{k},\right.
$$

holds for all $x, y \in A$.

Then $\operatorname{Bpp}(\mathrm{T})$ is nonempty.

\section{Remark 3.15.}

(i) The results proved in Section 3 generalize the corresponding results of Suzuki [24], Hussain et al. [6] and many others. They also enrich and develop existing conclusions on coupled best proximity point $[11,23]$. Meanwhile some creative and developed techniques are used to the proofs of our results well.

(ii) If we choose some other function $\Theta$ and some more choices for the forms of $\alpha, M(x, y)$ or $M\left(x, y, x^{\prime}, y^{\prime}\right)$ in the results of the above sections, then more fruitful results will be obtained.

\section{Acknowledgment}

This work is supported by China Postdoctoral Science Foundation (Grant No. 2014M551168, No. 2016T90244), Natural Science Foundation of Heilongjiang Province of China (Grant No. A201410).

\section{References}

[1] A. Abkar, M. Gabeleh, Best proximity points for cyclic mappings in ordered metric spaces, J. Optim. Theory Appl., 151 (2011), 418-424. 3.1

[2] C. Di Bari, T. Suzuki, C. Vetro, Best proximity points for cyclic Meir-Keeler contractions, Nonlinear Anal., 69 (2008), 3790-3794. 1

[3] A. A. Eldred, W. A. Kirk, P. Veeramani, Proximal normal structure and relatively nonexpansive mappings, Studia Math., 171 (2005), 283-293.

[4] A. A. Eldred, P. Veeramani, Existence and convergence of best proximity points, J. Math. Anal. Appl., 323 (2006), 1001-1006. 1

[5] K. Fan, Extensions of two fixed point theorems of F. E. Browder, Math. Z., 112 (1969), 234-240. 1

[6] N. Hussain, M. Hezarjaribi, M. A. Kutbi, P. Salimi, Best proximity results for Suzuki and convex type contractions, Fixed Point Theory Appl., 2016 (2016), 20 pages. 1, 2, 2.1, 2.3, 2, 2.4, 3.15 
[7] M. Jleli, B. Samet, A new generalization of the Banach contraction principle, J. Inequal. Appl., 2014 (2014), 8 pages. 1, $2,3,3.9$

[8] M. Jleli, B. Samet, Remarks on the paper: Best proximity point theorems: an exploration of a common solution to approximation and optimization problems, Appl. Math. Comput., 228 (2014), 366-370. 1

[9] A. Latif, M. Eshaghi Gordji, E. Karapınar, W. Sintunavarat, Fixed point results for generalized $(\alpha, \psi)-$ Meir-Keeler contractive mappings and applications, J. Inequal. Appl., 2014 (2014), 11 pages. 1, 2

[10] C. Mongkolkeha, P. Kumam, Best proximity point theorems for generalized cyclic contractions in ordered metric spaces, J. Optim. Theory Appl., 155 (2012), 215-226. 1

[11] J. Nantadilok, Coupled best proximity point theorems for $\alpha-\psi$-proximal contractive multimaps, Fixed Point Theory Appl., 2015 (2015), 14 pages. 1, 3.1, 3.4, 3.5, 3.15

[12] V. S. Raj, A best proximity point theorem for weakly contractive non-self-mappings, Nonlinear Anal., 74 (2011), $4804-$ 4808. 2, 2.2

[13] N. Redjel, A. Dehici, E. Karapınar, İ. M. Erhan, Fixed point theorems for $(\alpha, \psi)$-Meir-Keeler-Khan mappings, J. Nonlinear Sci. Appl., 8 (2015), 955-964. 1

[14] S. Reich, Approximate selections, best approximations, fixed points, and invariant sets, J. Math. Anal. Appl., 62 (1978), 104-113. 1

[15] S. Sadiq Basha, Best proximity points: global optimal approximate solutions, J. Global Optim., 49 (2011), $15-21$.

[16] S. Sadiq Basha, Best proximity point theorems, J. Approx. Theory, 163 (2011), 1772-1781.

[17] S. Sadiq Basha, Best proximity point theorems: an exploration of a common solution to approximation and optimization problems, Appl. Math. Comput., 218 (2012), 9773-9780.

[18] S. Sadiq Basha, N. Shahzad, R. Jeyaraj, Common best proximity points: global optimization of multi-objective functions, Appl. Math. Lett., 24 (2011), 883-886.

[19] S. Sadiq Basha, P. Veeramani, Best proximity pair theorems for multifunctions with open fibres, J. Approx. Theory, 103 (2000), 119-129. 1

[20] B. Samet, C. Vetro, P. Vetro, Fixed point theorems for $\alpha \psi$-contractive type mappings, Nonlinear Anal., 75 (2012), 2154-2165. 1

[21] V. M. Sehgal, S. P. Singh, A generalization to multifunctions of Fan's best approximation theorem, Proc. Amer. Math. Soc., 102 (1988), 534-537. 1

[22] V. M. Sehgal, S. P. Singh, A theorem on best approximations, Numer. Funct. Anal. Optim., 10 (1989), 181-184. 1

[23] W. Sintunavarat, P. Kumam, Coupled best proximity point theorem in metric spaces, Fixed Point Theory Appl., 2012 (2012), 16 pages. $1,3.15$

[24] T. Suzuki, The existence of best proximity points with the weak P-property, Fixed Point Theory Appl., 2013 (2013), 6 pages. $1,3.15$

[25] Z.-G. Wang, H.-L. Li, Fixed point theorems and endpoint theorems for $(\alpha, \psi)$-Meir-Keeler-Khan multivalued mappings, Fixed Point Theory Appl., 2016 (2016), 18 pages. 1

[26] K. Włodarczyk, R. Plebaniak, A. Banach, Best proximity points for cyclic and noncyclic set-valued relatively quasiasymptotic contractions in uniform spaces, Nonlinear Anal., 70 (2009), 3332-3341. 1 